\title{
Cobalt clusters with cubane-type topologies based on trivacant polyoxometalate ligands
}

Yan Duan, Juan M. Clemente-Juan, Carlos Giménez-Saiz*, Eugenio Coronado*

Supporting Information 


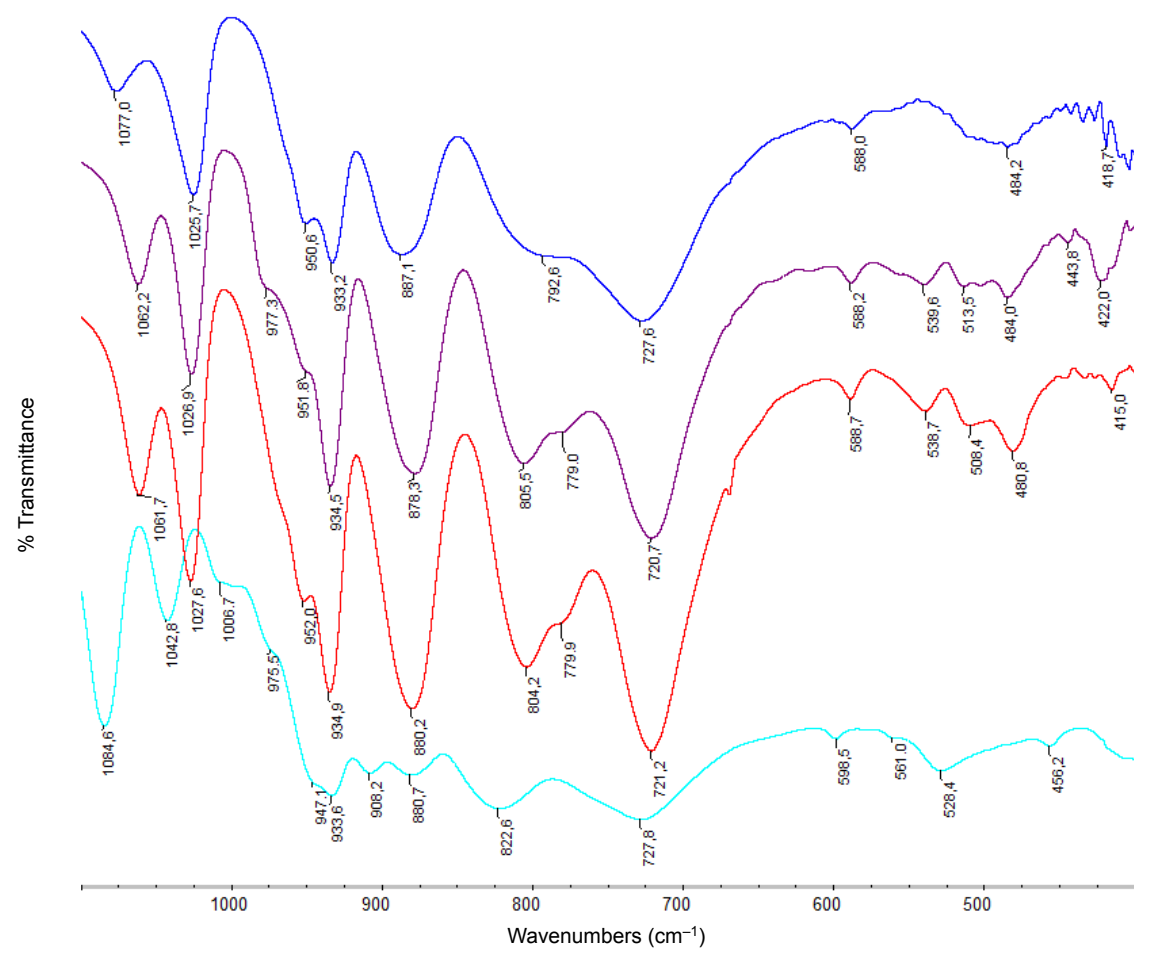

Figure S1. FT-IR spectra of compounds $\mathrm{Na}_{1.5} \mathrm{Cs}_{2.5}-\mathbf{1}$ (blue), $\mathbf{C s}_{\mathbf{8}} \mathbf{K}-\mathbf{2}$ (violet), $\mathbf{K}_{5} \mathbf{N a}_{2}-\mathbf{3}$ (red) and $\mathrm{Na}_{15}-\mathbf{4}$ (cyan).

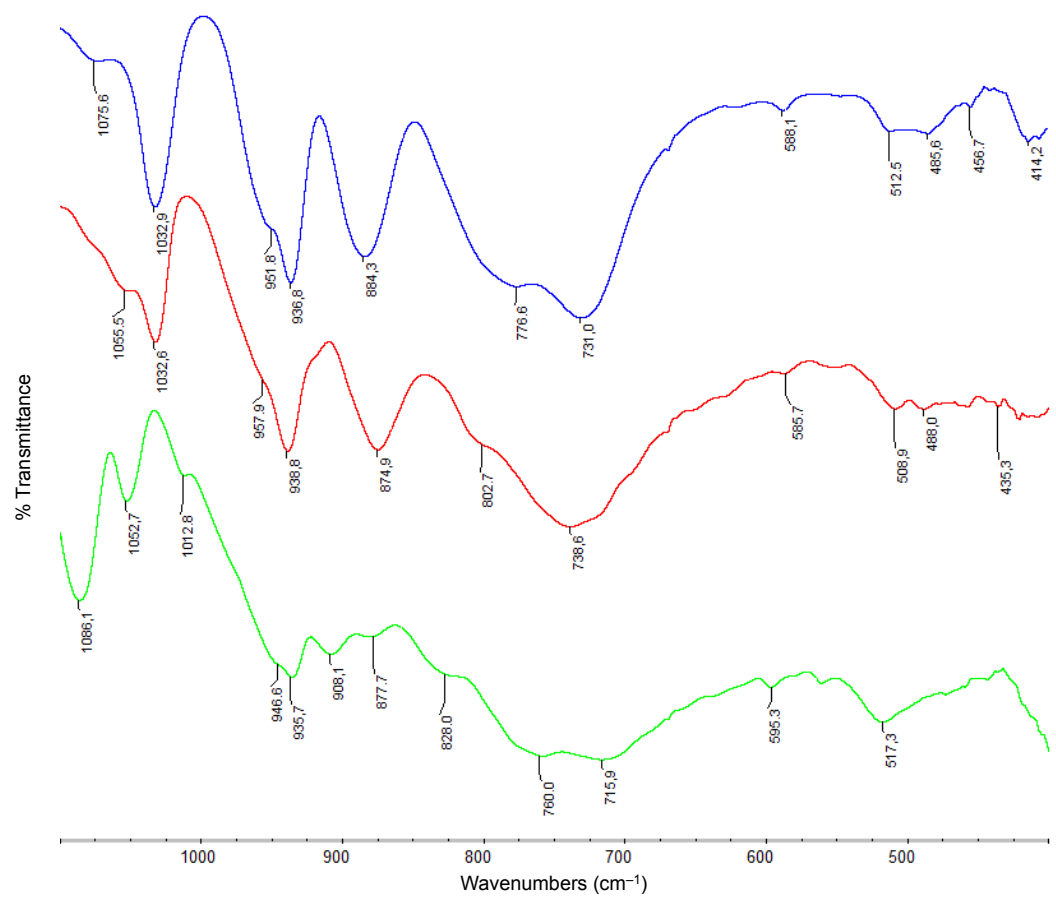

Figure S2. FT-IR spectra of the precipitates obtained when $\mathrm{CsCl}$ is added during the synthesis of $\mathbf{N a}_{\mathbf{1 . 5}} \mathbf{C s}_{2.5}-\mathbf{1}$ (blue), $\mathbf{K}_{5} \mathbf{N a}_{\mathbf{2}}-\mathbf{3}$ (red), and $\mathrm{Na}_{15}-\mathbf{4}$ (cyan) which allow their identification as mixed cessium/sodium salts of the tetracobalt sandwich compounds $\left[\mathrm{Co}_{4}{ }_{4}\left(\mathrm{H}_{2} \mathrm{O}\right)_{2}\left(\mathrm{PW}_{9} \mathrm{O}_{34}\right)_{2}\right]^{10-},\left[\left(\mathrm{Co}^{\mathrm{II}} / \mathrm{Co}^{\mathrm{III}}\right)_{4}\left(\mathrm{H}_{2} \mathrm{O}\right)_{2}\left(\mathrm{PW}_{9} \mathrm{O}_{34}\right)_{2}\right]^{\mathrm{n}-}$ and $\left[\mathrm{Co}_{4}\left(\mathrm{H}_{2} \mathrm{O}\right)_{2}\left(\mathrm{P}_{2} \mathrm{~W}_{15} \mathrm{O}_{56}\right)_{2}\right]^{16-}$ respectively, by comparison with the spectra of the original potassium or sodium salts of these polyanions (Finke, R. G.; Droege, M. W.; Domaille P. J. Inorg. Chem. 1987, 26, 38863896). 


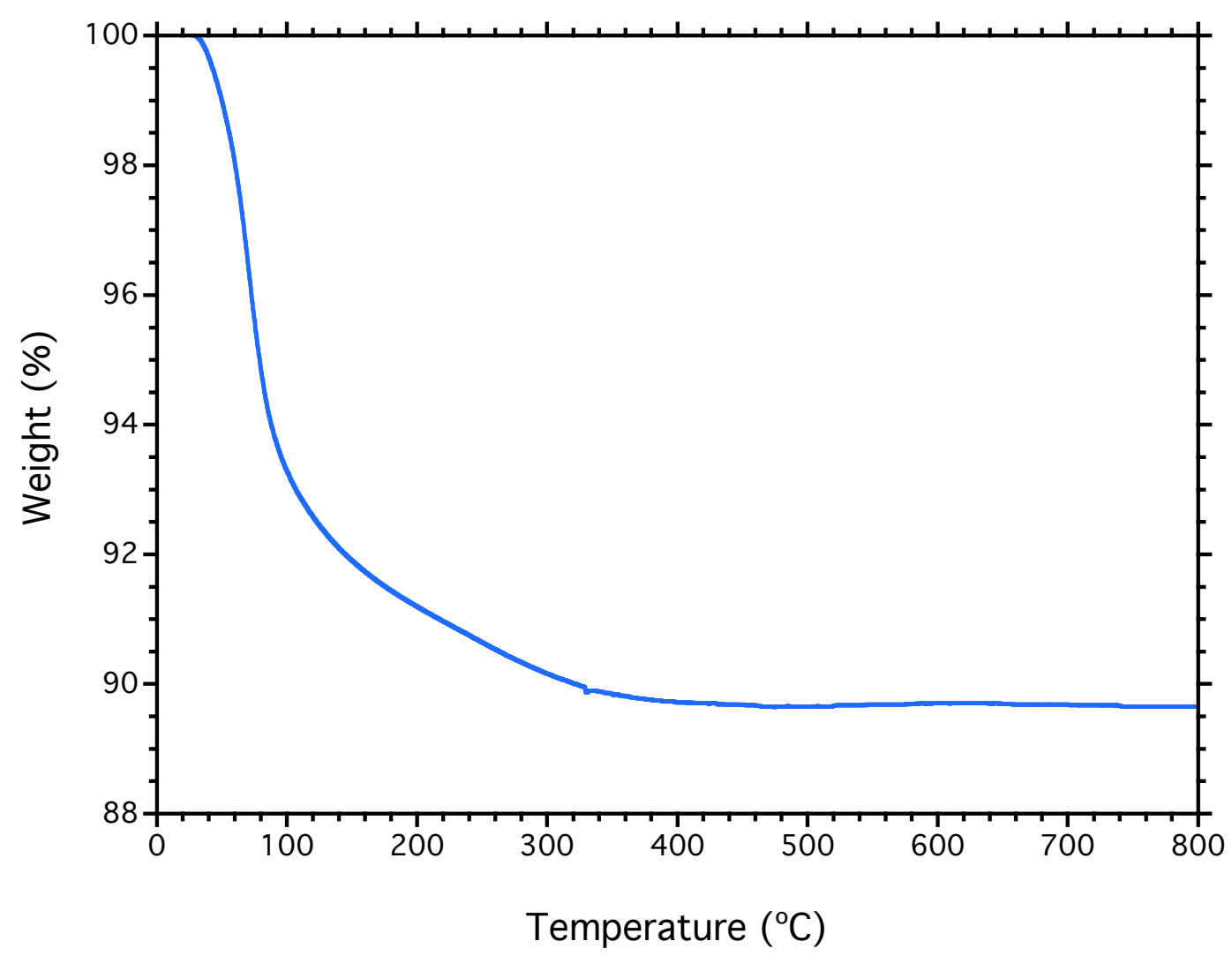

Figure S3. Thermogram of $\mathbf{N a}_{1.5} \mathbf{C s}_{2.5} \mathbf{- 1}$ from room temperature to $800{ }^{\circ} \mathrm{C}$.

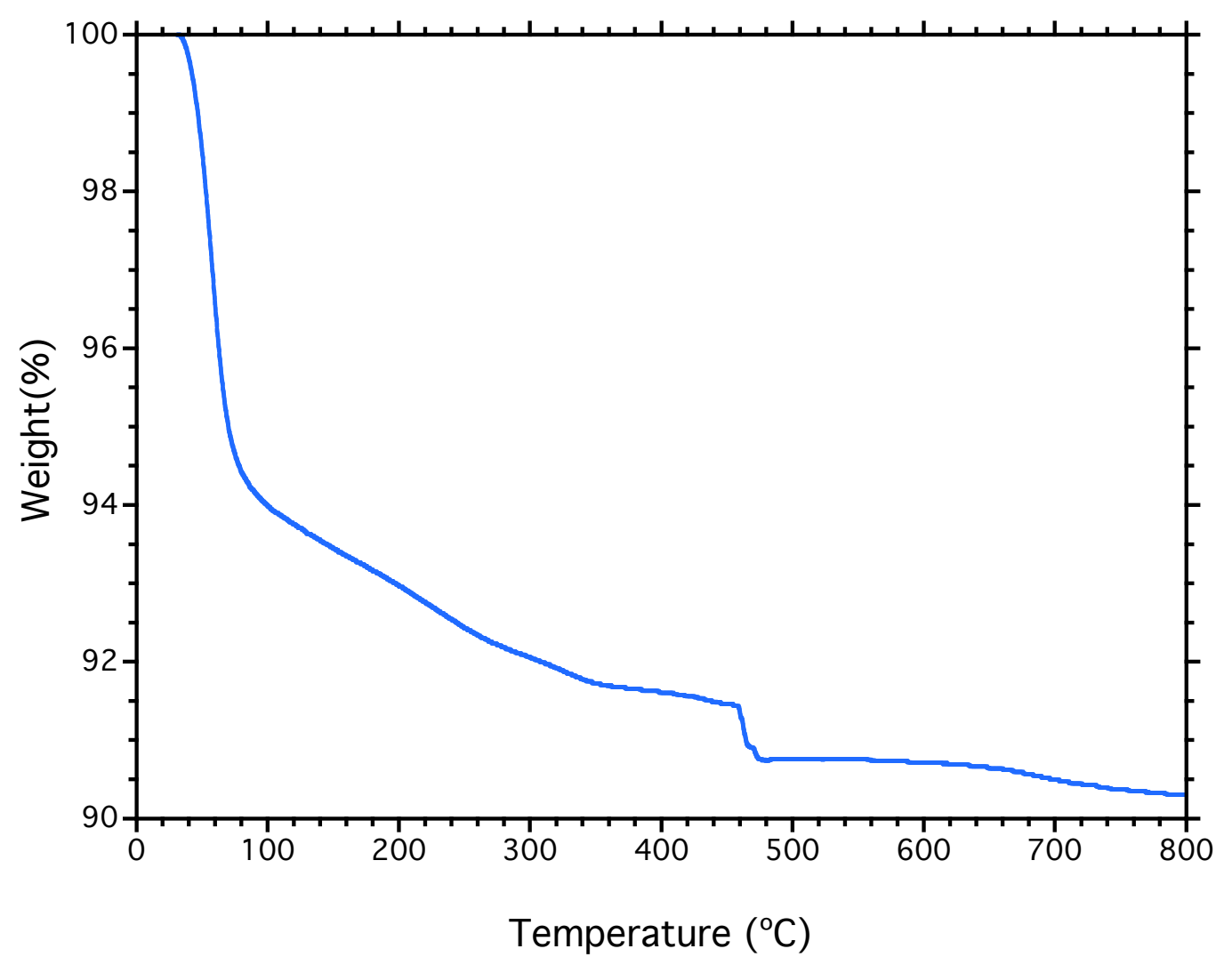

Figure S4. Thermogram of $\mathbf{C s}_{7} \mathbf{N a}_{2}-\mathbf{2}$ from room temperature to $800{ }^{\circ} \mathrm{C}$. 


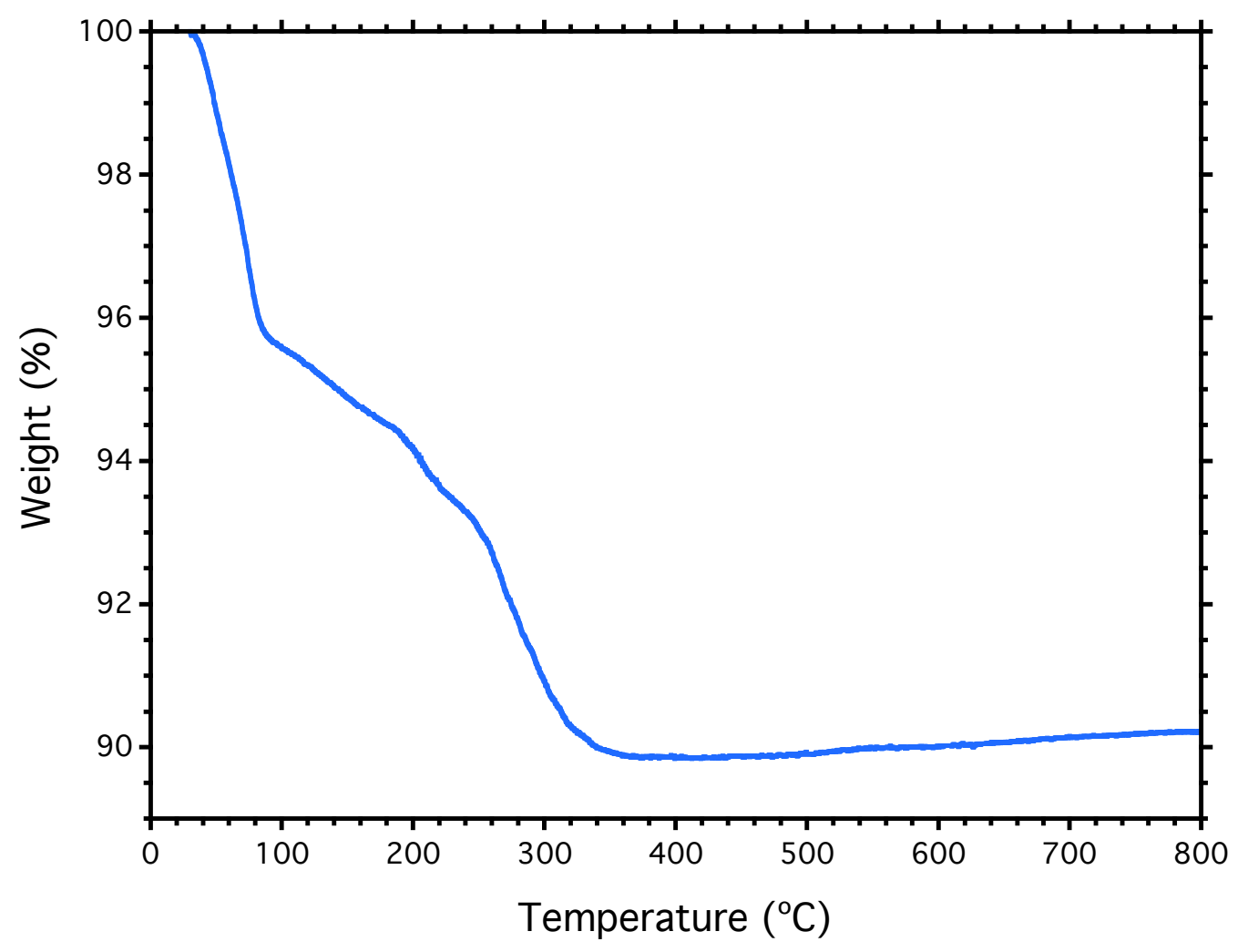

Figure S5. Thermogram of $\mathbf{K}_{\mathbf{5}} \mathbf{N a}_{\mathbf{2}} \mathbf{- 3}$ from room temperature to $800{ }^{\circ} \mathrm{C}$.

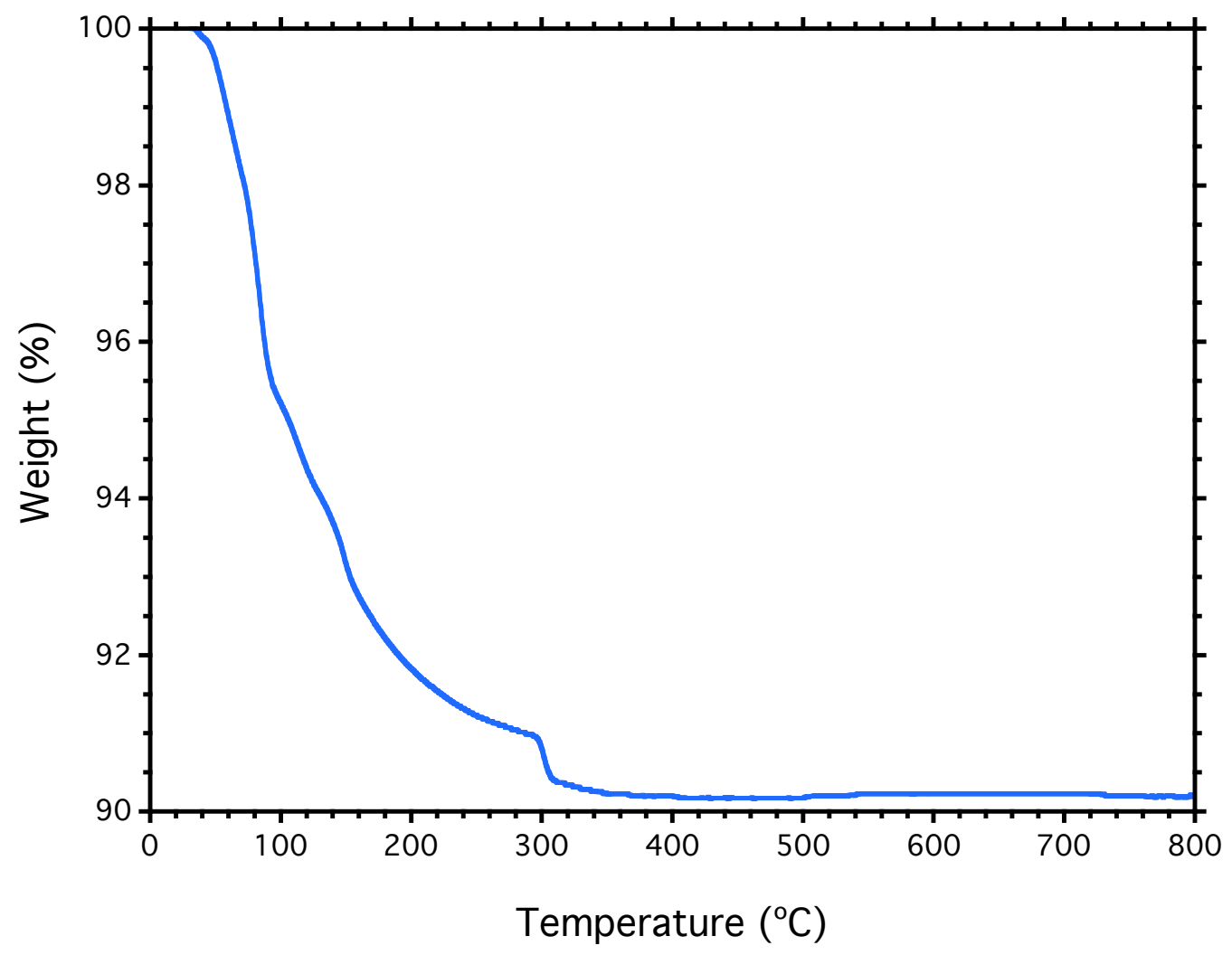

Figure S6. Thermogram of $\mathbf{N a}_{\mathbf{1 5}} \mathbf{- 4}$ from room temperature to $800{ }^{\circ} \mathrm{C}$. 
Table S1. Bond Distances $(\AA)$ and Angles $\left(^{\circ}\right)$ involved in the Cubane and di-Cubane Clusters contained in POMs 1, 2, 3 and 4.

\begin{tabular}{|c|c|c|c|c|}
\hline & 1 & 2 & 3 & 4 \\
\hline $\mathrm{Co}(1)-\mathrm{O}(1)\left[\mathrm{O}(5)^{i}\right]^{a}$ & $2.134(10)$ & 1.91(3) & $1.915(10)$ & $1.931(9)$ \\
\hline $\mathrm{Co}(1)-\mathrm{O}(2)\left[\mathrm{O}(6)^{i}\right]^{a}$ & $2.162(9)$ & $1.93(3)$ & $1.917(10)$ & $1.913(9)$ \\
\hline $\mathrm{Co}(1)-\mathrm{O}(3)\left[\mathrm{O}(4)^{i}\right]^{a}$ & $2.060(11)$ & $1.92(3)$ & $1.912(11)$ & $1.912(9)$ \\
\hline $\mathrm{Co}(1)-\mathrm{O}(4)$ & $2.080(9)$ & $1.92(3)$ & $1.912(11)$ & $1.912(9)$ \\
\hline $\mathrm{Co}(1)-\mathrm{O}(5)$ & $2.113(10)$ & $1.91(3)$ & $1.915(10)$ & $1.931(9)$ \\
\hline $\mathrm{Co}(1)-\mathrm{O}(6)$ & $2.083(9)$ & $1.93(3)$ & $1.917(10)$ & $1.913(9)$ \\
\hline $\mathrm{Co}(2)-\mathrm{O}(4)$ & $2.089(9)$ & $2.06(3)$ & $2.092(11)$ & $2.080(9)$ \\
\hline $\mathrm{Co}(2)-\mathrm{O}(6)$ & $2.060(9)$ & $2.06(3)$ & $2.096(11)$ & $2.093(8)$ \\
\hline $\mathrm{Co}(2)-\mathrm{O}(7)$ & $2.139(9)$ & $2.13(3)$ & $2.163(11)$ & $2.131(10)$ \\
\hline $\mathrm{Co}(2)-\mathrm{O}(10)$ & $2.262(9)$ & $2.25(3)$ & $2.254(9)$ & $2.179(8)$ \\
\hline $\mathrm{Co}(2)-\mathrm{O}(11)$ & $2.031(8)$ & $1.97(3)$ & $1.991(11)$ & $2.011(8)$ \\
\hline $\mathrm{Co}(2)-\mathrm{O}(16)$ & $2.053(10)$ & $2.04(3)$ & $2.009(10)$ & $2.011(9)$ \\
\hline $\mathrm{Co}(3)-\mathrm{O}(4)$ & $2.070(9)$ & $2.11(3)$ & $2.082(10)$ & $2.094(9)$ \\
\hline $\mathrm{Co}(3)-\mathrm{O}(5)$ & $2.062(10)$ & $2.09(2)$ & $2.087(11)$ & $2.085(9)$ \\
\hline $\mathrm{Co}(3)-\mathrm{O}(8)$ & $2.141(11)$ & $2.13(4)$ & $2.139(11)$ & $2.118(11)$ \\
\hline $\mathrm{Co}(3)-\mathrm{O}(10)$ & $2.269(8)$ & $2.24(2)$ & $2.263(10)$ & $2.174(8)$ \\
\hline $\mathrm{Co}(3)-\mathrm{O}(12)$ & $2.072(10)$ & $2.08(3)$ & $2.030(11)$ & $2.012(8)$ \\
\hline $\mathrm{Co}(3)-\mathrm{O}(13)$ & $2.052(10)$ & $2.09(3)$ & $1.991(11)$ & $2.026(8)$ \\
\hline $\mathrm{Co}(4)-\mathrm{O}(5)$ & $2.043(9)$ & $2.06(3)$ & $2.069(10)$ & $2.102(9)$ \\
\hline $\mathrm{Co}(4)-\mathrm{O}(6)$ & $2.023(9)$ & $2.07(3)$ & $2.080(10)$ & $2.077(9)$ \\
\hline Co(4)-O(9) & $2.127(10)$ & $2.21(3)$ & $2.100(10)$ & $2.108(10)$ \\
\hline $\mathrm{Co}(4)-\mathrm{O}(10)$ & $2.261(9)$ & $2.29(2)$ & $2.232(10)$ & $2.189(8)$ \\
\hline $\mathrm{Co}(4)-\mathrm{O}(14)$ & $2.025(10)$ & $2.01(3)$ & $1.986(10)$ & $2.015(8)$ \\
\hline $\mathrm{Co}(4)-\mathrm{O}(15)$ & $2.053(9)$ & $1.98(3)$ & $1.984(11)$ & $2.010(9)$ \\
\hline $\mathrm{O}(4)-\mathrm{Co}(1)-\mathrm{O}(5)$ & $78.6(4)$ & $86.8(11)$ & $84.3(4)$ & $84.3(4)$ \\
\hline $\mathrm{O}(4)-\mathrm{Co}(1)-\mathrm{O}(6)$ & $80.6(3)$ & $82.5(13)$ & $85.1(5)$ & $85.1(4)$ \\
\hline $\mathrm{O}(5)-\mathrm{Co}(1)-\mathrm{O}(6)$ & 78.4(4) & $84.2(11)$ & $86.4(4)$ & $86.0(4)$ \\
\hline $\mathrm{O}(4)-\mathrm{Co}(1)-\mathrm{O}(3)\left[\mathrm{O}(4)^{i}\right]^{a}$ & 175.1(4) & $180.0(17)$ & $180.0(2)$ & $180.000(1)$ \\
\hline $\mathrm{O}(4)-\mathrm{Co}(1)-\mathrm{O}(1)\left[\mathrm{O}(5)^{i}\right]^{a}$ & $91.2(3)$ & $93.2(11)$ & $95.7(4)$ & $95.7(4)$ \\
\hline $\mathrm{O}(4)-\mathrm{Co}(1)-\mathrm{O}(2)\left[\mathrm{O}(6)^{i}\right]^{a}$ & $95.6(4)$ & $97.5(13)$ & $94.9(5)$ & $94.9(4)$ \\
\hline $\mathrm{O}(5)-\mathrm{Co}(1)-\mathrm{O}(3)\left[\mathrm{O}(4)^{i}\right]^{a}$ & $97.8(4)$ & $93.2(11)$ & 95.7(4) & $95.7(4)$ \\
\hline $\mathrm{O}(5)-\mathrm{Co}(1)-\mathrm{O}(1)\left[\mathrm{O}(5)^{i}\right]^{a}$ & 168.4(4) & $180.0(14)$ & $180.000(1)$ & $180.000(2)$ \\
\hline $\mathrm{O}(5)-\mathrm{Co}(1)-\mathrm{O}(2)\left[\mathrm{O}(6)^{i}\right]^{a}$ & $97.6(4)$ & $95.8(11)$ & $93.6(4)$ & $94.0(4)$ \\
\hline $\mathrm{O}(6)-\mathrm{Co}(1)-\mathrm{O}(3)\left[\mathrm{O}(4)^{i}\right]^{a}$ & $95.4(4)$ & $97.5(13)$ & $94.9(5)$ & $94.9(4)$ \\
\hline $\mathrm{O}(6)-\mathrm{Co}(1)-\mathrm{O}(1)\left[\mathrm{O}(5)^{i}\right]^{a}$ & $94.6(4)$ & $95.8(11)$ & 93.6(4) & $94.0(4)$ \\
\hline $\mathrm{O}(6)-\mathrm{Co}(1)-\mathrm{O}(2)\left[\mathrm{O}(6)^{i}\right]^{a}$ & 174.9(4) & $180.0(18)$ & $180.000(1)$ & $180.000(2)$ \\
\hline $\mathrm{O}(3)\left[\mathrm{O}(4)^{i}\right]-\mathrm{Co}(1)-\mathrm{O}(1)\left[\mathrm{O}(5)^{i}\right]^{a}$ & $92.1(4)$ & $86.8(11)$ & $84.3(4)$ & $84.3(4)$ \\
\hline $\mathrm{O}(3)\left[\mathrm{O}(4)^{i}\right]-\mathrm{Co}(1)-\mathrm{O}(2)\left[\mathrm{O}(6)^{i}\right]^{a}$ & $88.2(4)$ & $82.5(13)$ & $85.1(5)$ & $85.1(4)$ \\
\hline $\mathrm{O}(1)\left[\mathrm{O}(5)^{i}\right]-\mathrm{Co}(1)-\mathrm{O}(2)\left[\mathrm{O}(6)^{i}\right]^{a}$ & $88.8(4)$ & $84.2(11)$ & $86.4(4)$ & $86.0(4)$ \\
\hline $\mathrm{O}(11)-\mathrm{Co}(2)-\mathrm{O}(16)$ & $85.8(4)$ & $85.6(13)$ & $88.0(4)$ & $90.5(4)$ \\
\hline $\mathrm{O}(11)-\mathrm{Co}(2)-\mathrm{O}(6)$ & $172.7(4)$ & $169.2(14)$ & $173.5(4)$ & $171.2(4)$ \\
\hline $\mathrm{O}(16)-\mathrm{Co}(2)-\mathrm{O}(6)$ & $97.1(4)$ & $99.6(13)$ & $95.5(4)$ & $95.5(4)$ \\
\hline $\mathrm{O}(11)-\mathrm{Co}(2)-\mathrm{O}(4)$ & $95.6(4)$ & $97.4(13)$ & $99.5(5)$ & $96.9(4)$ \\
\hline $\mathrm{O}(16)-\mathrm{Co}(2)-\mathrm{O}(4)$ & 174.1(4) & $171.6(13)$ & $169.5(4)$ & $170.8(3)$ \\
\hline $\mathrm{O}(6)-\mathrm{Co}(2)-\mathrm{O}(4)$ & $80.9(3)$ & $76.2(13)$ & 76.4(4) & $76.6(4)$ \\
\hline $\mathrm{O}(11)-\mathrm{Co}(2)-\mathrm{O}(7)$ & $94.2(4)$ & $98.8(13)$ & $93.8(4)$ & $93.1(4)$ \\
\hline $\mathrm{O}(16)-\mathrm{Co}(2)-\mathrm{O}(7)$ & $90.8(4)$ & $100.2(13)$ & $99.0(4)$ & $88.8(4)$ \\
\hline $\mathrm{O}(6)-\mathrm{Co}(2)-\mathrm{O}(7)$ & $92.5(4)$ & $89.6(11)$ & 91.1(4) & $93.5(4)$ \\
\hline $\mathrm{O}(4)-\mathrm{Co}(2)-\mathrm{O}(7)$ & $94.9(4)$ & $87.0(12)$ & $87.9(4)$ & $96.3(4)$ \\
\hline $\mathrm{O}(11)-\mathrm{Co}(2)-\mathrm{O}(10)$ & $91.3(3)$ & $89.4(12)$ & $93.8(4)$ & $91.9(3)$ \\
\hline $\mathrm{O}(16)-\mathrm{Co}(2)-\mathrm{O}(10)$ & $91.8(3)$ & $89.8(11)$ & $90.8(4)$ & $92.9(3)$ \\
\hline
\end{tabular}




\begin{tabular}{|c|c|c|c|c|}
\hline & 1 & 2 & 3 & 4 \\
\hline $\mathrm{O}(6)-\mathrm{Co}(2)-\mathrm{O}(10)$ & $81.9(3)$ & $81.2(9)$ & $80.7(4)$ & $81.3(3)$ \\
\hline $\mathrm{O}(4)-\mathrm{Co}(2)-\mathrm{O}(10)$ & $82.3(3)$ & $82.5(10)$ & $81.4(4)$ & $81.4(3)$ \\
\hline $\mathrm{O}(7)-\mathrm{Co}(2)-\mathrm{O}(10)$ & $174.0(4)$ & $167.5(10)$ & $167.8(4)$ & $174.7(4)$ \\
\hline $\mathrm{O}(13)-\mathrm{Co}(3)-\mathrm{O}(12)$ & $86.6(4)$ & $91.3(10)$ & $89.0(4)$ & $90.1(3)$ \\
\hline $\mathrm{O}(13)-\mathrm{Co}(3)-\mathrm{O}(5)$ & $96.2(4)$ & $92.0(10)$ & $97.6(4)$ & $95.9(3)$ \\
\hline $\mathrm{O}(12)-\mathrm{Co}(3)-\mathrm{O}(5)$ & $172.4(4)$ & $172.4(11)$ & $170.4(4)$ & $171.1(4)$ \\
\hline $\mathrm{O}(13)-\mathrm{Co}(3)-\mathrm{O}(4)$ & $171.5(4)$ & $167.2(12)$ & $172.4(5)$ & $169.7(3)$ \\
\hline $\mathrm{O}(12)-\mathrm{Co}(3)-\mathrm{O}(4)$ & $96.3(4)$ & $98.2(11)$ & $96.7(4)$ & $97.1(3)$ \\
\hline $\mathrm{O}(5)-\mathrm{Co}(3)-\mathrm{O}(4)$ & $80.0(4)$ & $77.6(11)$ & $76.1(4)$ & $76.2(3)$ \\
\hline $\mathrm{O}(13)-\mathrm{Co}(3)-\mathrm{O}(8)$ & $87.2(4)$ & $99.6(14)$ & $94.7(4)$ & $93.0(4)$ \\
\hline $\mathrm{O}(12)-\mathrm{Co}(3)-\mathrm{O}(8)$ & $97.1(4)$ & $88.5(11)$ & $92.6(4)$ & $89.3(4)$ \\
\hline $\mathrm{O}(5)-\mathrm{Co}(3)-\mathrm{O}(8)$ & $90.1(4)$ & $97.6(10)$ & $93.6(4)$ & $96.9(4)$ \\
\hline $\mathrm{O}(4)-\mathrm{Co}(3)-\mathrm{O}(8)$ & $100.4(5)$ & $89.2(13)$ & $90.0(4)$ & $94.5(4)$ \\
\hline $\mathrm{O}(13)-\mathrm{Co}(3)-\mathrm{O}(10)$ & $89.4(3)$ & $89.7(12)$ & $93.5(4)$ & $91.3(3)$ \\
\hline $\mathrm{O}(12)-\mathrm{Co}(3)-\mathrm{O}(10)$ & $90.8(3)$ & $92.1(9)$ & $92.4(4)$ & $91.1(3)$ \\
\hline $\mathrm{O}(5)-\mathrm{Co}(3)-\mathrm{O}(10)$ & $82.2(3)$ & $81.2(9)$ & $80.4(4)$ & $82.2(3)$ \\
\hline $\mathrm{O}(4)-\mathrm{Co}(3)-\mathrm{O}(10)$ & $82.6(3)$ & $81.5(11)$ & $81.4(4)$ & $81.2(3)$ \\
\hline $\mathrm{O}(8)-\mathrm{Co}(3)-\mathrm{O}(10)$ & $171.2(4)$ & $170.6(13)$ & $170.5(4)$ & $175.7(4)$ \\
\hline $\mathrm{O}(14)-\mathrm{Co}(4)-\mathrm{O}(6)$ & $172.3(4)$ & $171.6(14)$ & $168.2(5)$ & $171.9(4)$ \\
\hline $\mathrm{O}(14)-\mathrm{Co}(4)-\mathrm{O}(5)$ & $96.6(4)$ & $96.2(12)$ & $94.2(4)$ & $96.4(3)$ \\
\hline $\mathrm{O}(6)-\mathrm{Co}(4)-\mathrm{O}(5)$ & $81.4(4)$ & $77.1(12)$ & $78.5(4)$ & $77.7(4)$ \\
\hline $\mathrm{O}(14)-\mathrm{Co}(4)-\mathrm{O}(15)$ & $86.5(4)$ & $87.7(14)$ & $89.6(4)$ & $90.8(4)$ \\
\hline $\mathrm{O}(6)-\mathrm{Co}(4)-\mathrm{O}(15)$ & $94.5(4)$ & $97.9(15)$ & $96.5(4)$ & $94.5(4)$ \\
\hline $\mathrm{O}(5)-\mathrm{Co}(4)-\mathrm{O}(15)$ & $171.5(4)$ & $169.4(11)$ & $171.8(4)$ & $170.5(3)$ \\
\hline $\mathrm{O}(14)-\mathrm{Co}(4)-\mathrm{O}(9)$ & $92.3(4)$ & $93.1(17)$ & $97.3(4)$ & $90.7(4)$ \\
\hline $\mathrm{O}(6)-\mathrm{Co}(4)-\mathrm{O}(9)$ & $95.4(4)$ & $92.7(17)$ & $91.7(4)$ & $95.3(4)$ \\
\hline $\mathrm{O}(5)-\mathrm{Co}(4)-\mathrm{O}(9)$ & $98.8(4)$ & $94.4(13)$ & $88.4(4)$ & $95.0(4)$ \\
\hline $\mathrm{O}(15)-\mathrm{Co}(4)-\mathrm{O}(9)$ & $89.0(4)$ & $95.2(13)$ & $98.2(5)$ & $91.1(4)$ \\
\hline $\mathrm{O}(14)-\mathrm{Co}(4)-\mathrm{O}(10)$ & $89.6(3)$ & $93.7(11)$ & $88.2(4)$ & $92.2(3)$ \\
\hline $\mathrm{O}(6)-\mathrm{Co}(4)-\mathrm{O}(10)$ & $82.7(3)$ & $80.1(11)$ & $81.6(4)$ & $81.5(3)$ \\
\hline $\mathrm{O}(5)-\mathrm{Co}(4)-\mathrm{O}(10)$ & $82.8(3)$ & $80.8(9)$ & $81.5(4)$ & $81.5(3)$ \\
\hline $\mathrm{O}(15)-\mathrm{Co}(4)-\mathrm{O}(10)$ & $89.2(3)$ & $89.2(10)$ & $91.4(4)$ & $92.0(3)$ \\
\hline $\mathrm{O}(9)-\mathrm{Co}(4)-\mathrm{O}(10)$ & $177.3(4)$ & $172.0(15)$ & $168.8(4)$ & $175.7(4)$ \\
\hline $\mathrm{Co}(1)-\mathrm{O}(4)-\mathrm{Co}(2)$ & $97.2(4)$ & $100.3(14)$ & $98.7(5)$ & $98.8(4)$ \\
\hline $\mathrm{Co}(3)-\mathrm{O}(4)-\mathrm{Co}(1)$ & $100.0(4)$ & $96.3(11)$ & $99.3(5)$ & $99.5(4)$ \\
\hline $\mathrm{Co}(3)-\mathrm{O}(4)-\mathrm{Co}(2)$ & $102.9(4)$ & $102.4(13)$ & $102.9(4)$ & $100.6(4)$ \\
\hline $\mathrm{Co}(3)-\mathrm{O}(5)-\mathrm{Co}(1)$ & $99.2(4)$ & $97.2(12)$ & $99.0(5)$ & $99.2(4)$ \\
\hline $\mathrm{Co}(4)-\mathrm{O}(5)-\mathrm{Co}(1)$ & $98.1(4)$ & $98.6(11)$ & $96.8(4)$ & $96.8(4)$ \\
\hline $\mathrm{Co}(4)-\mathrm{O}(5)-\mathrm{Co}(3)$ & $103.6(4)$ & $104.4(11)$ & $103.8(5)$ & $100.2(4)$ \\
\hline $\mathrm{Co}(2)-\mathrm{O}(6)-\mathrm{Co}(1)$ & $98.0(3)$ & $100.0(17)$ & $98.4(5)$ & $98.3(4)$ \\
\hline $\mathrm{Co}(4)-\mathrm{O}(6)-\mathrm{Co}(2)$ & $104.2(4)$ & $105.1(12)$ & $103.3(5)$ & $101.1(4)$ \\
\hline $\mathrm{Co}(4)-\mathrm{O}(6)-\mathrm{Co}(1)$ & $99.7(4)$ & $97.9(11)$ & $96.4(4)$ & $98.2(4)$ \\
\hline $\mathrm{Co}(2)-\mathrm{O}(10)-\mathrm{Co}(3)$ & $91.7(3)$ & $92.5(9)$ & $92.5(3)$ & $95.1(3)$ \\
\hline $\mathrm{Co}(2)-\mathrm{O}(10)-\mathrm{Co}(4)$ & $90.9(3)$ & $92.3(10)$ & $93.7(3)$ & $94.9(3)$ \\
\hline $\mathrm{Co}(3)-\mathrm{O}(10)-\mathrm{Co}(4)$ & $90.8(3)$ & $92.9(8)$ & $93.3(4)$ & $94.8(3)$ \\
\hline
\end{tabular}

${ }^{a}$ For compounds $\mathbf{2 , 3}$ and $\mathbf{4}$ the atom labels enclosed between square brackets must be used instead the preceding atom label. Symmetry transformations: (i) $1-\mathrm{x},-\mathrm{y},-\mathrm{z}$ (for 2 ); $1-\mathrm{x},-\mathrm{y}$, $1+\mathrm{z}($ for 3) and 1-x, 1-y, 1-z (for 4). 


\section{Bond valence sum (BVS) calculations:}

The determination of the oxidation states of metal centers and the protonation states of oxygen sites, bond valence sum (BVS) calculations were carried out using the method of I. D. Brown ${ }^{1}$. The calculation formula is $S_{i}=\exp \left[\left(R_{0}-R_{i}\right) / B\right]$, where $S_{i}$ is the bond valence of bond $i, \mathrm{R}_{0}$ is a constant dependent upon the bonded elements, $\mathrm{R}_{i}$ is the bond length of bond $i$, and $B$ equals to $0.37 . R_{0}$ values were taken from the literature for calculations performed on cobalt $^{2}$ and oxygen ${ }^{2,3}$ sites $\left[\mathrm{R}_{0}(\mathrm{Co}-\mathrm{O})=1.67\right]$.

[1] Brown, I. D.; Altermatt, D. Acta Crystallogr. Sect. B, 1985, 41, 244-247.

[2] Wood, R. M.; Palenik, G. J. Inorg. Chem. 1998, 37, 4149-4151.

[3] Brese, N. E.; O’Keeffe, M. Acta Crystallogr. Sect. B, 1991, 47, 192-197.

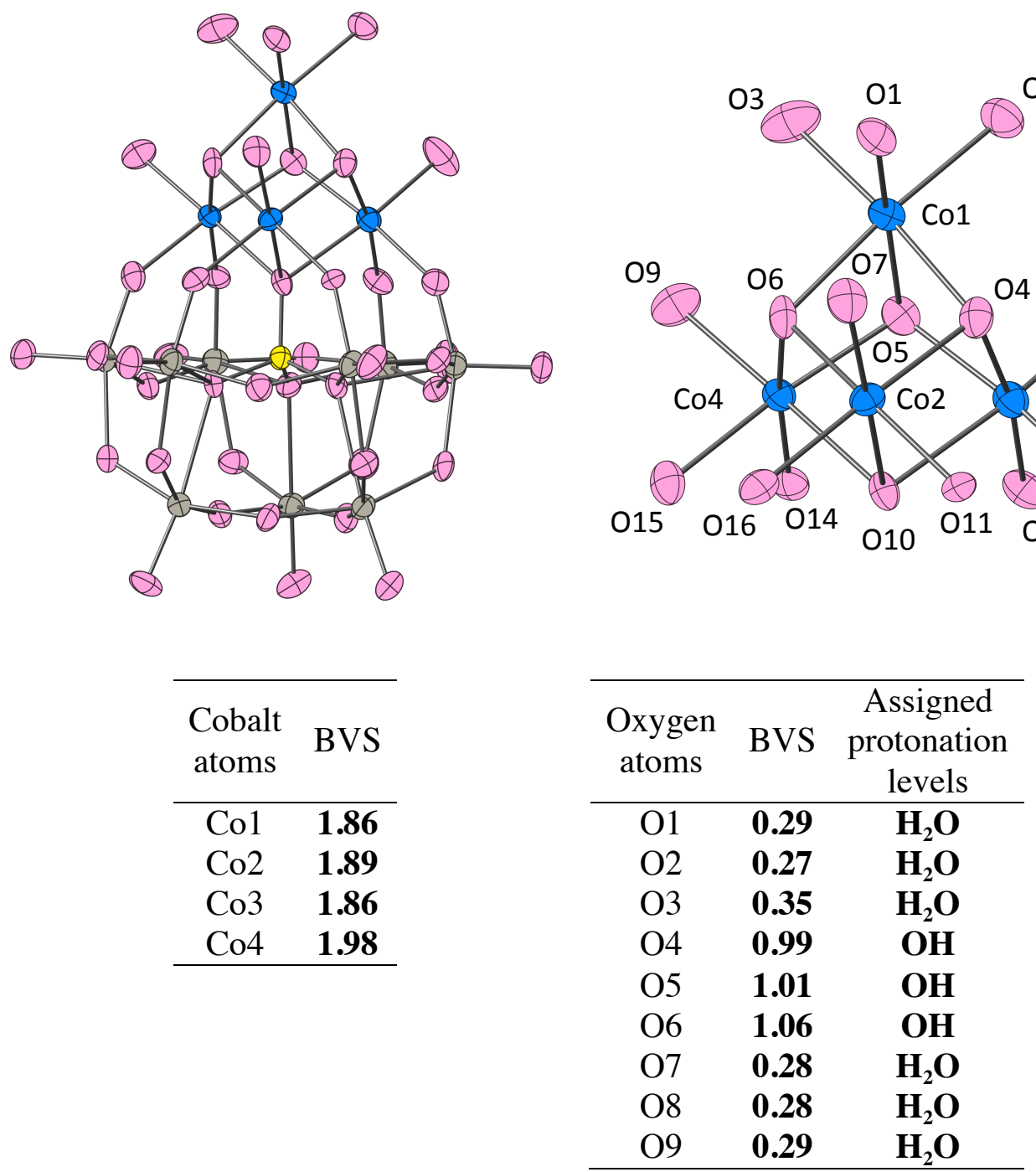

Figure S7. Bond valence sum (BVS) calculations for cobalt centers and relevant oxygen sites of polyoxometalate $\mathbf{1}$. 

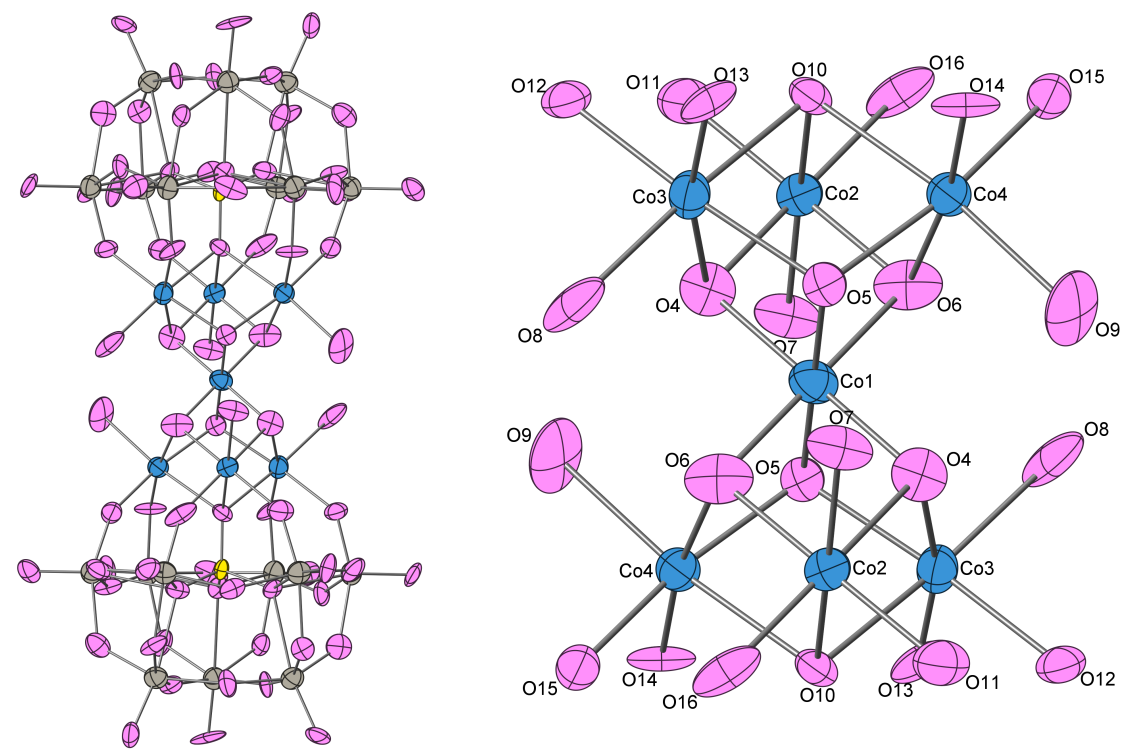

\begin{tabular}{cc}
\hline $\begin{array}{c}\text { Cobalt } \\
\text { atoms }\end{array}$ & BVS \\
\hline Co1 & $\mathbf{3 . 0 6}$ \\
Co 2 & $\mathbf{2 . 0 1}$ \\
Co 3 & $\mathbf{1 . 7 7}$ \\
Co 4 & $\mathbf{1 . 9 3}$ \\
\hline
\end{tabular}

\begin{tabular}{ccc}
\hline $\begin{array}{c}\text { Oxygen } \\
\text { atoms }\end{array}$ & BVS & $\begin{array}{c}\text { Assigned } \\
\text { protonation } \\
\text { levels }\end{array}$ \\
\hline O4 & $\mathbf{1 . 1 7}$ & $\mathbf{O H}$ \\
O5 & $\mathbf{1 . 2 3}$ & $\mathbf{O H}$ \\
O6 & $\mathbf{1 . 2 3}$ & $\mathbf{O H}$ \\
O7 & $\mathbf{0 . 2 9}$ & $\mathbf{H}_{\mathbf{2}} \mathbf{O}$ \\
O8 & $\mathbf{0 . 2 8}$ & $\mathbf{H}_{\mathbf{2}} \mathbf{O}$ \\
O9 & $\mathbf{0 . 2 3}$ & $\mathbf{H}_{\mathbf{2}} \mathbf{O}$ \\
O10 & $\mathbf{1 . 8 6}$ & $\mathbf{O}$ \\
\hline
\end{tabular}

Figure S8. Bond valence sum (BVS) calculations for cobalt centers and relevant oxygen sites of polyoxometalate 2 . 


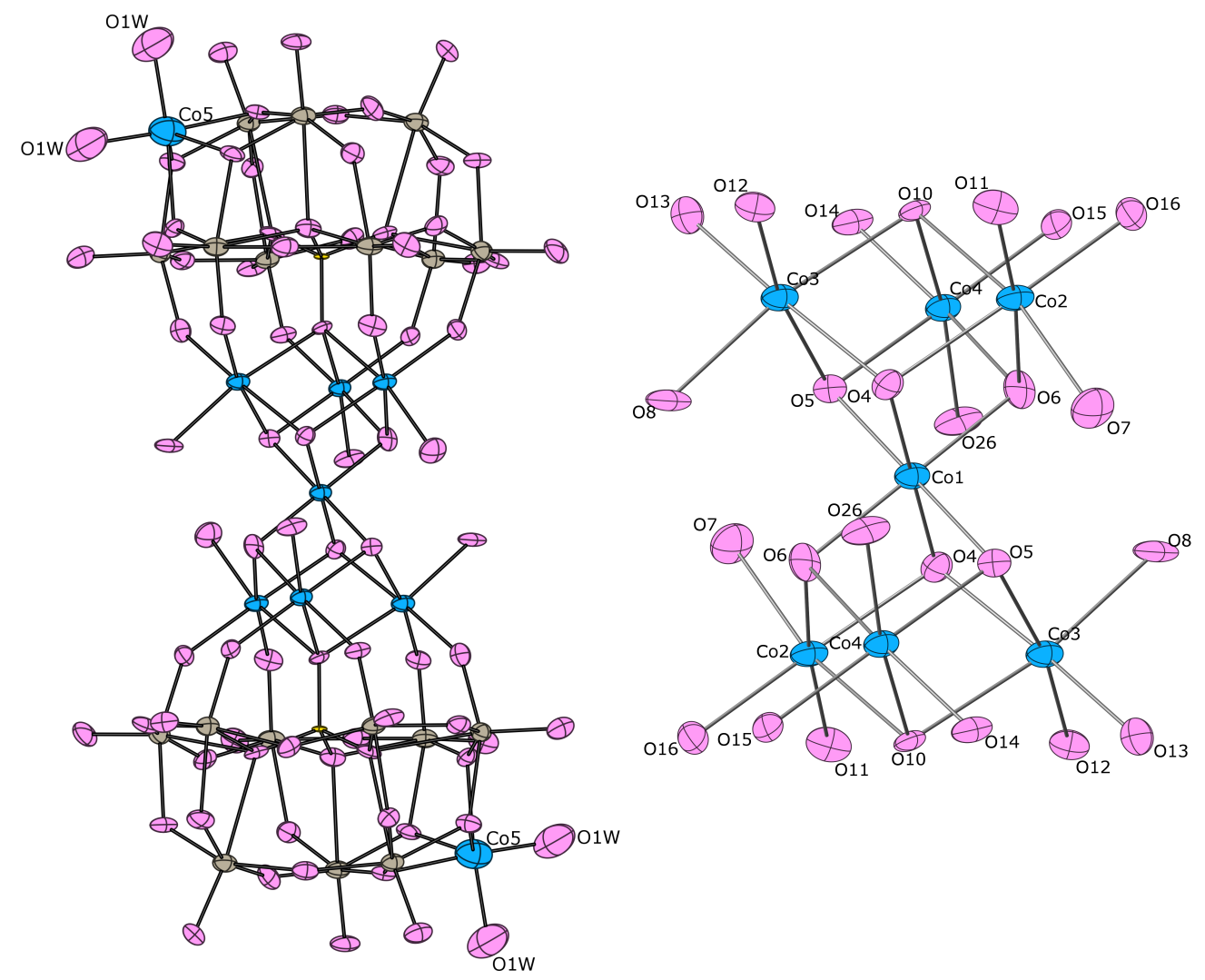

\begin{tabular}{cc}
\hline $\begin{array}{c}\text { Cobalt } \\
\text { atoms }\end{array}$ & BVS \\
\hline Co1 & $\mathbf{3 . 0 9}$ \\
Co 2 & $\mathbf{1 . 9 3}$ \\
Co 3 & $\mathbf{1 . 9 3}$ \\
Co 4 & $\mathbf{2 . 0 6}$ \\
Co5 & $\mathbf{2 . 0 6}$ \\
\hline
\end{tabular}

\begin{tabular}{ccc}
\hline $\begin{array}{c}\text { Oxygen } \\
\text { atoms }\end{array}$ & BVS & $\begin{array}{c}\text { Assigned } \\
\text { protonation } \\
\text { levels }\end{array}$ \\
\hline O4 & $\mathbf{1 . 1 7}$ & $\mathbf{O H}$ \\
O5 & $\mathbf{1 . 1 8}$ & $\mathbf{O H}$ \\
O6 & $\mathbf{1 . 1 6}$ & $\mathbf{O H}$ \\
O7 & $\mathbf{0 . 2 6}$ & $\mathbf{H}_{\mathbf{2}}$ \\
O8 & $\mathbf{0 . 2 8}$ & $\mathbf{H}_{\mathbf{2}} \mathbf{O}$ \\
O10 & $\mathbf{1 . 7 9}$ & $\mathbf{O}$ \\
O26 & $\mathbf{1 . 5 5}$ & $\mathbf{O}$ \\
\hline
\end{tabular}

Figure S9. Bond valence sum (BVS) calculations for cobalt centers and relevant oxygen sites of polyoxometalate 3 . 


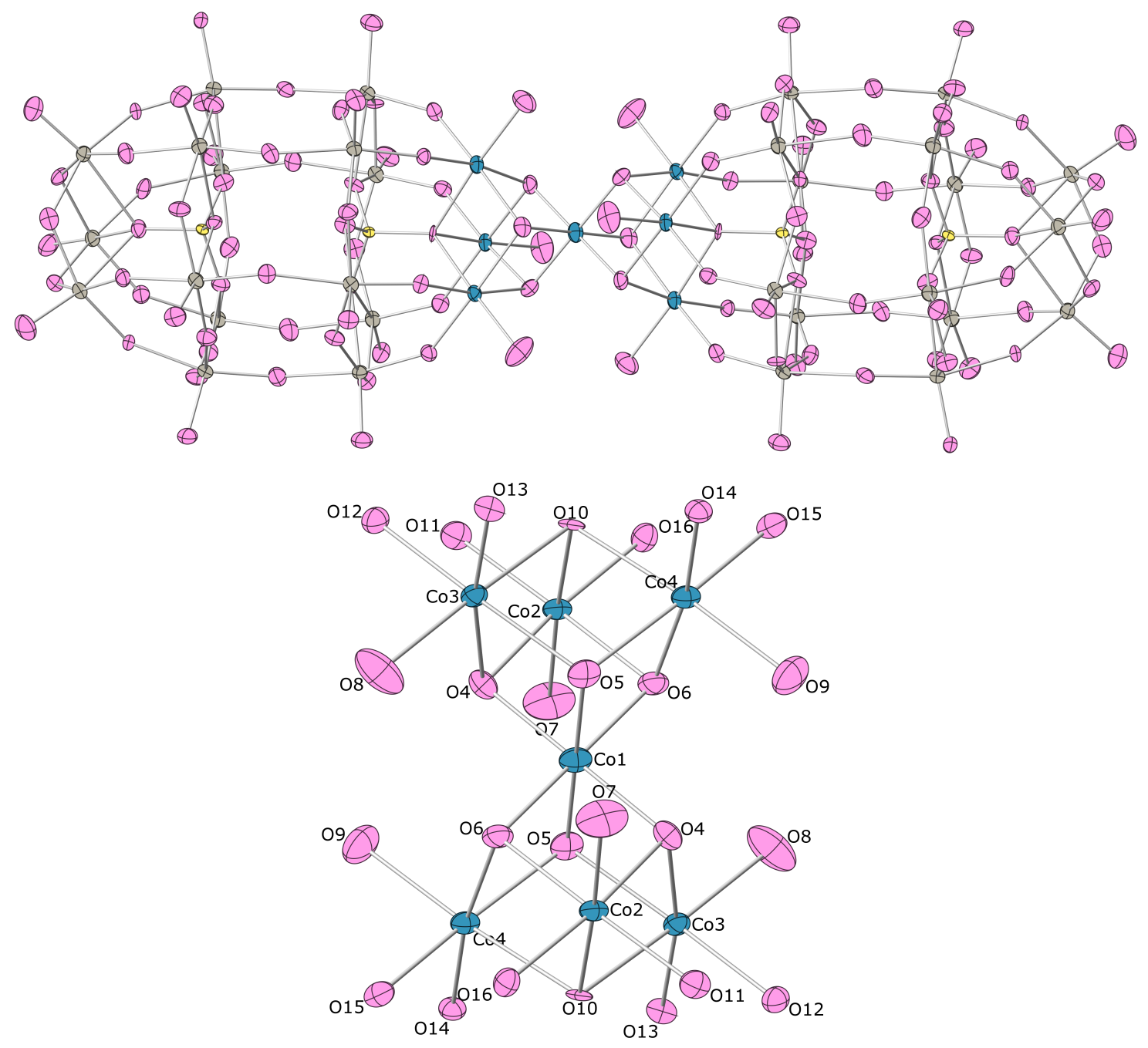

\begin{tabular}{cc}
\hline $\begin{array}{c}\text { Cobalt } \\
\text { atoms }\end{array}$ & BVS \\
\hline Co1 & $\mathbf{3 . 0 6}$ \\
Co2 & $\mathbf{1 . 9 7}$ \\
Co3 & $\mathbf{1 . 9 8}$ \\
Co 4 & $\mathbf{1 . 9 9}$ \\
\hline
\end{tabular}

\begin{tabular}{ccc}
\hline $\begin{array}{c}\text { Oxygen } \\
\text { atoms }\end{array}$ & BVS & $\begin{array}{c}\text { Assigned } \\
\text { protonation } \\
\text { levels }\end{array}$ \\
\hline O4 & $\mathbf{1 . 1 7}$ & $\mathbf{O H}$ \\
O5 & $\mathbf{1 . 1 7}$ & $\mathbf{O H}$ \\
O6 & $\mathbf{1 . 2 1}$ & $\mathbf{O H}$ \\
O7 & $\mathbf{0 . 2 9}$ & $\mathbf{H}_{2} \mathbf{O}$ \\
O8 & $\mathbf{0 . 3 0}$ & $\mathbf{H}_{2} \mathbf{O}$ \\
O9 & $\mathbf{0 . 3 1}$ & $\mathbf{H}_{2} \mathbf{O}$ \\
O10 & $\mathbf{1 . 8 4}$ & $\mathbf{O}$ \\
\hline
\end{tabular}

Figure S10. Bond valence sum (BVS) calculations for cobalt centers and relevant oxygen sites of polyoxometalate 4 . 

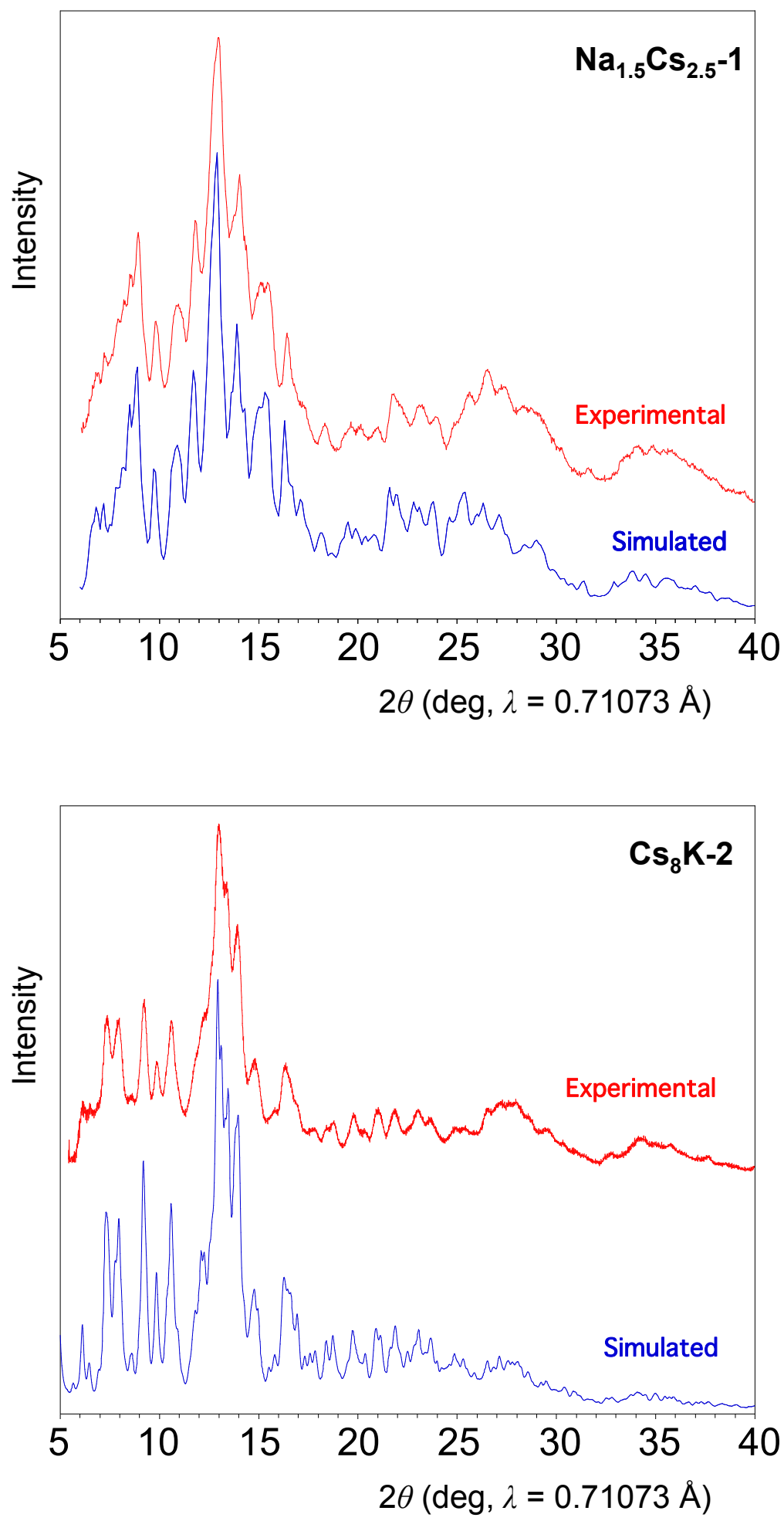

Figure S11. Comparison of simulated and experimental powder X-ray diffraction patterns for compounds $\mathbf{N a}_{1.5} \mathbf{C s}_{\mathbf{2 . 5}} \mathbf{- 1}$ (up) and $\mathbf{C s}_{\mathbf{8}} \mathbf{K}-\mathbf{2}$ (down). The experimental powder X-ray diffraction patterns were obtained from powdered samples in an Oxford Diffraction Supernova diffractometer. The simulated patterns were generated from the atomic coordinates of the single-crystal structure solutions using the program Mercury 3.0 (copyright CCDC, http://www.ccdc.cam.ac.uk/mercury/) and a FWHM (full width at half maximum) of 0.2. 

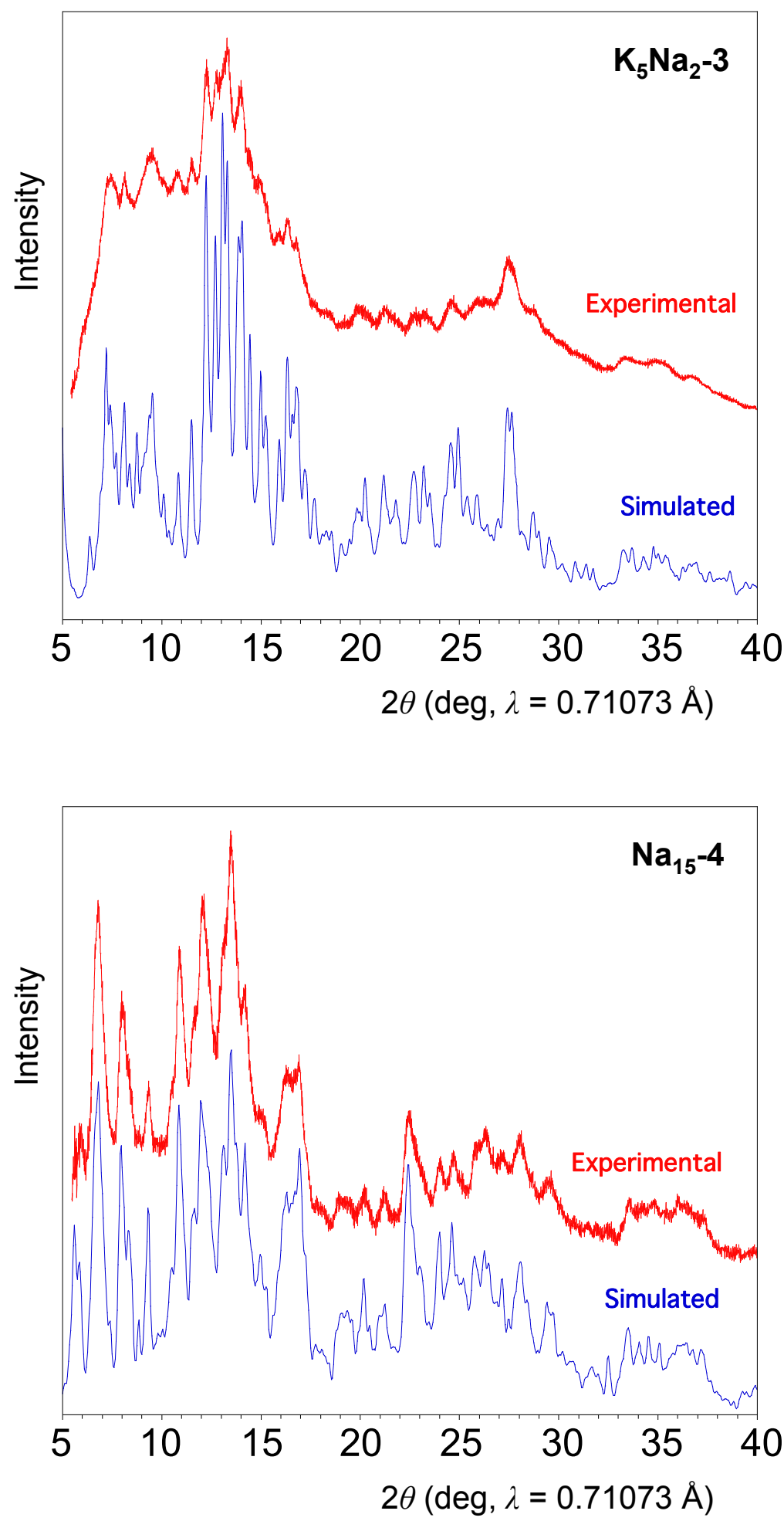

Figure S12. Comparison of simulated and experimental powder X-ray diffraction patterns for compounds $\mathbf{K}_{5} \mathbf{N a}_{2}-\mathbf{3}$ (up) and $\mathbf{N a}_{15}-\mathbf{4}$ (down). The experimental powder X-ray diffraction patterns were obtained from powdered samples in an Oxford Diffraction Supernova diffractometer. The simulated patterns were generated from the atomic coordinates of the single-crystal structure solutions using the program Mercury 3.0 (copyright CCDC, http://www.ccdc.cam.ac.uk/mercury/) and a FWHM (full width at half maximum) of 0.2. 


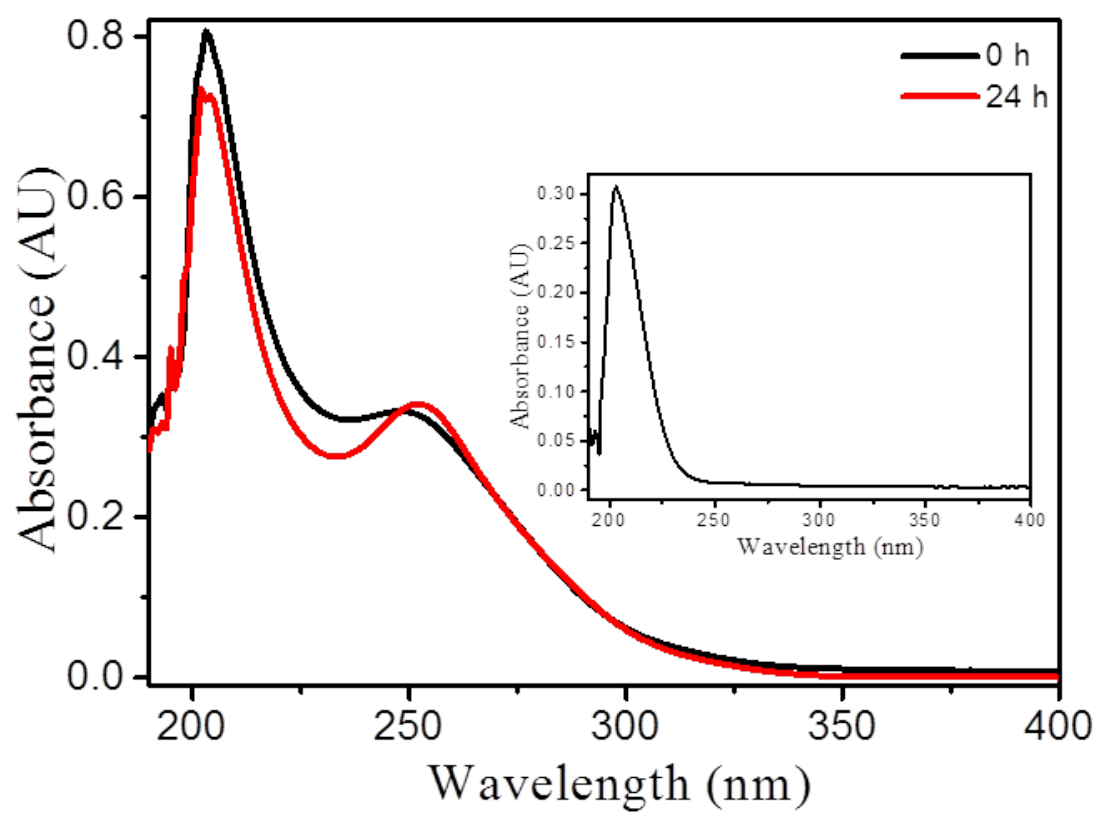

Figure S13. Evolution of the UV spectrum of a solution of POM $1\left(2.5 \times 10^{-6} \mathrm{M}\right)$ with time, recorded in a pH 5 medium (red and black lines). Inset: UV spectrum of a solution of $\mathrm{CoSO}_{4}$ $\left(7.5 \times 10^{-4} \mathrm{M}\right)$ in the same medium.

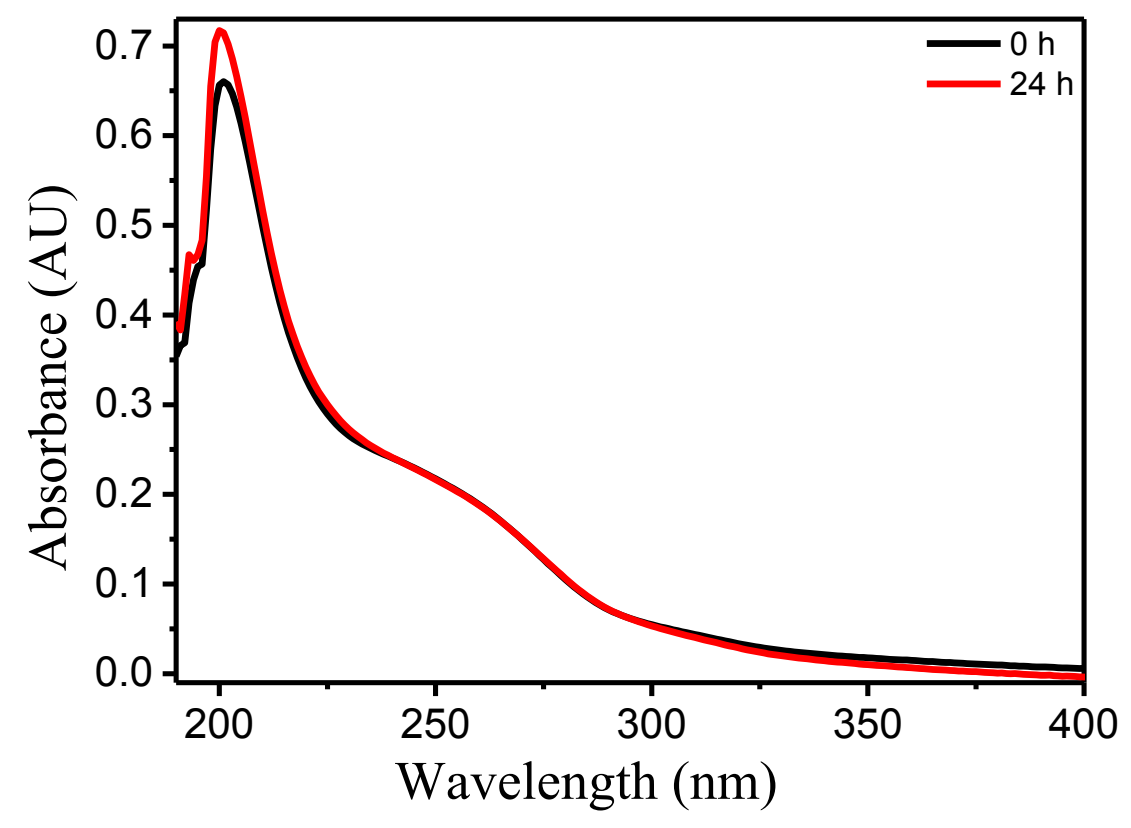

Figure S14. Evolution of the UV spectrum of a solution of POM $1\left(2.5 \times 10^{-6} \mathrm{M}\right)$ with time, recorded in a pH 9 medium (red and black lines). 


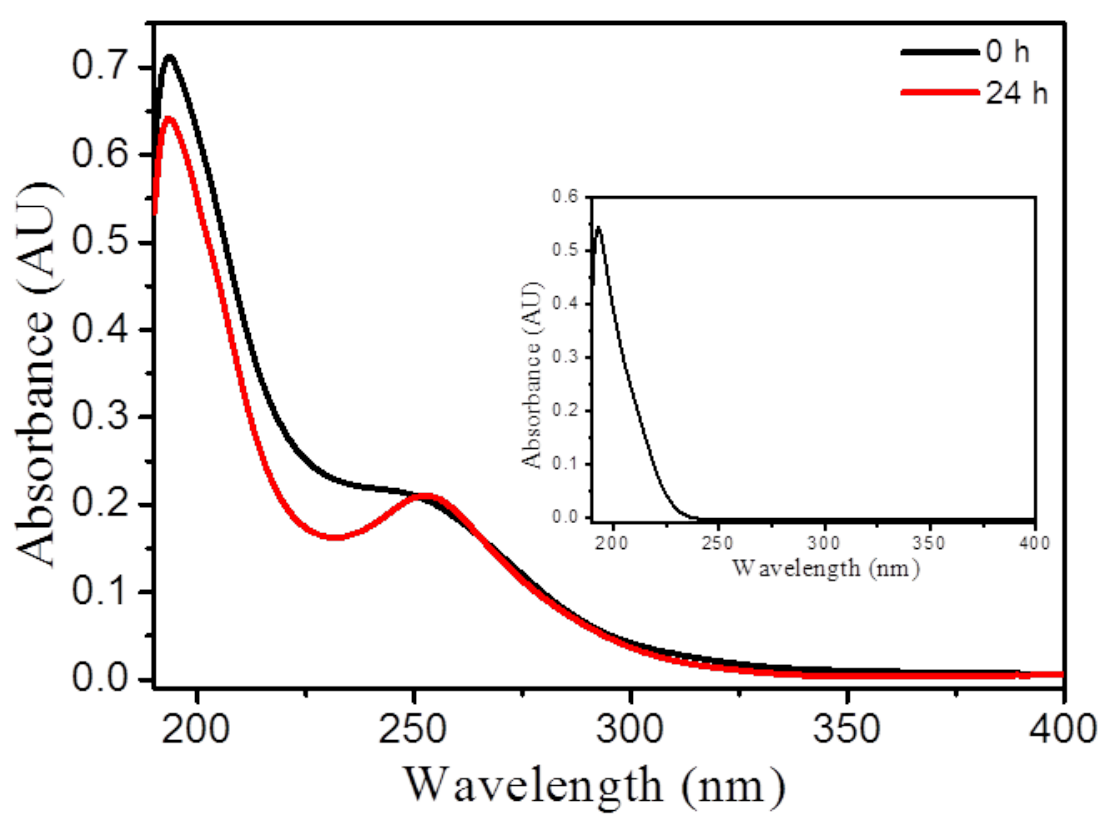

Figure S15. Evolution of the UV spectrum of a solution of POM $2\left(2.5 \times 10^{-6} \mathrm{M}\right)$ with time, recorded in a pH 3 medium (red and black lines). Inset: UV spectrum of a solution of $\mathrm{CoSO}_{4}$ $\left(7.5 \times 10^{-4} \mathrm{M}\right)$ in the same medium.

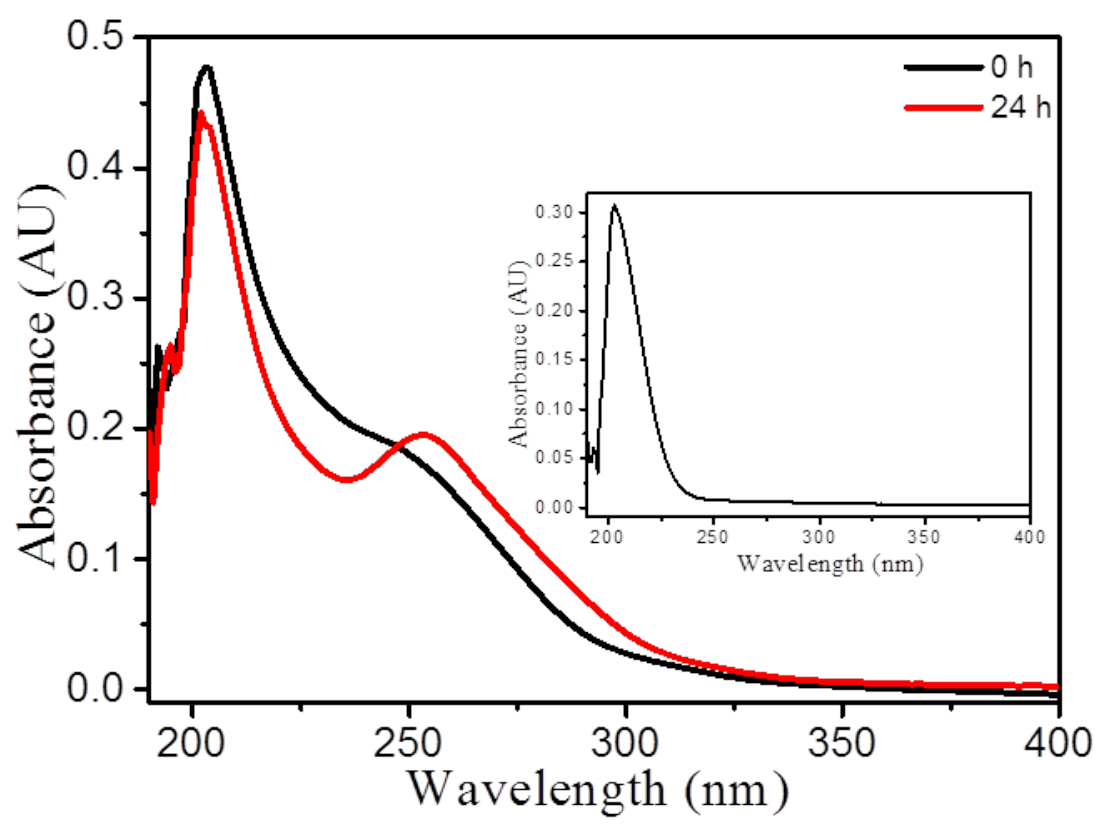

Figure S16. Evolution of the UV spectrum of a solution of POM $2\left(2.5 \times 10^{-6} \mathrm{M}\right)$ with time, recorded in a pH 5 medium (red and black lines). Inset: UV spectrum of a solution of $\mathrm{CoSO}_{4}$ $\left(7.5 \times 10^{-4} \mathrm{M}\right)$ in the same medium. 


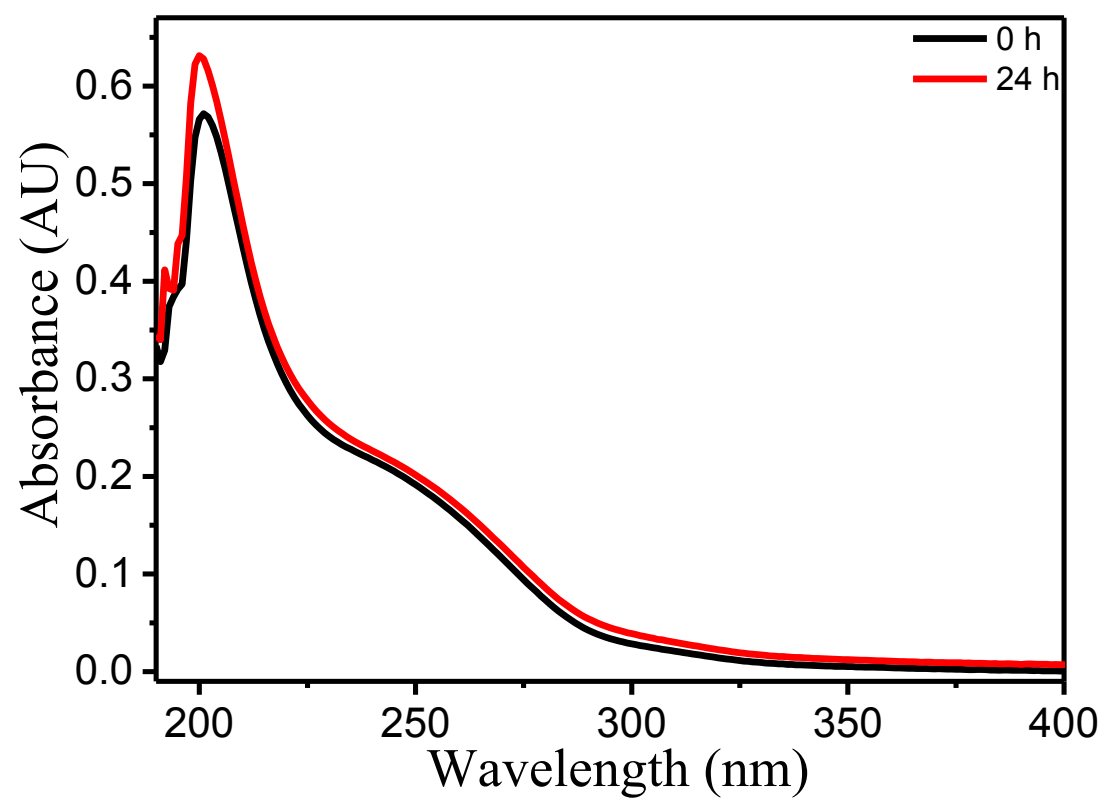

Figure S17. Evolution of the UV spectrum of a solution of POM $2\left(2.5 \times 10^{-6} \mathrm{M}\right)$ with time, recorded in a pH 9 medium (red and blue lines).

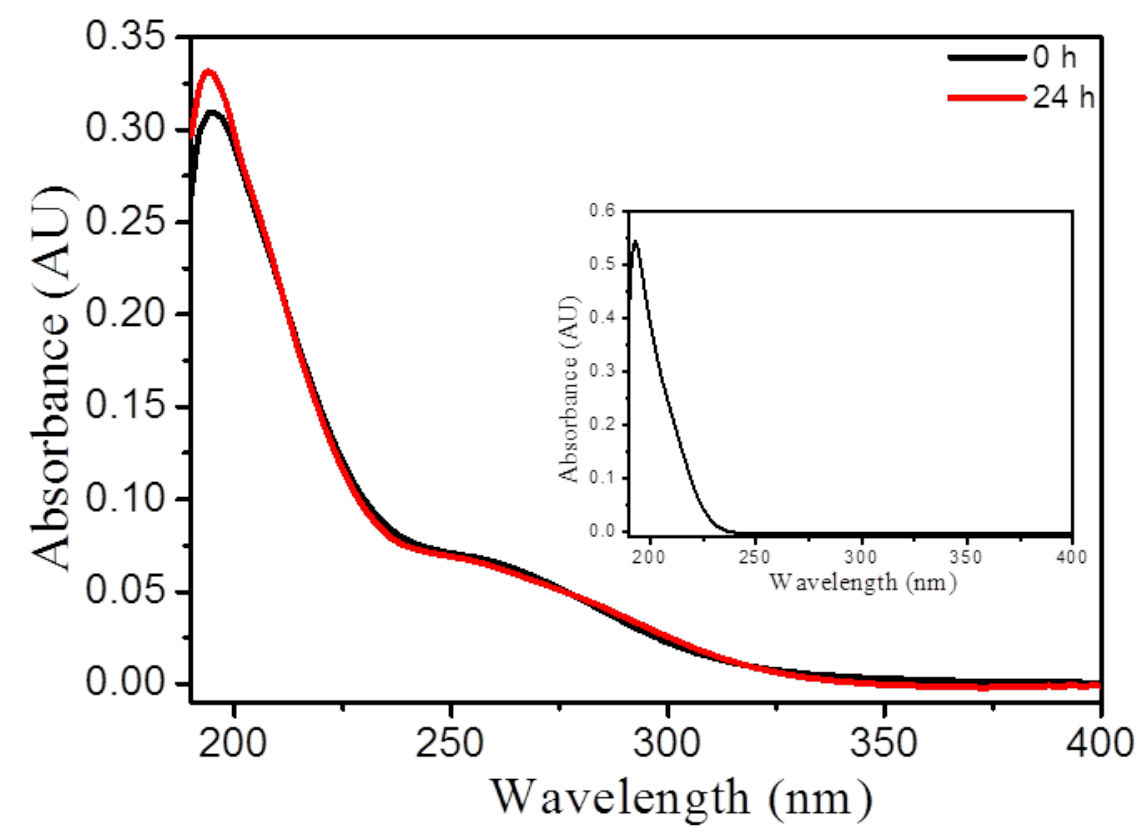

Figure S18. Evolution of the UV spectrum of a solution of POM $4\left(2.5 \times 10^{-6} \mathrm{M}\right)$ with time, recorded in a pH 3 medium (red and black lines). Inset: UV spectrum of a solution of $\mathrm{CoSO}_{4}$ $\left(7.5 \times 10^{-4} \mathrm{M}\right)$ in the same medium. 


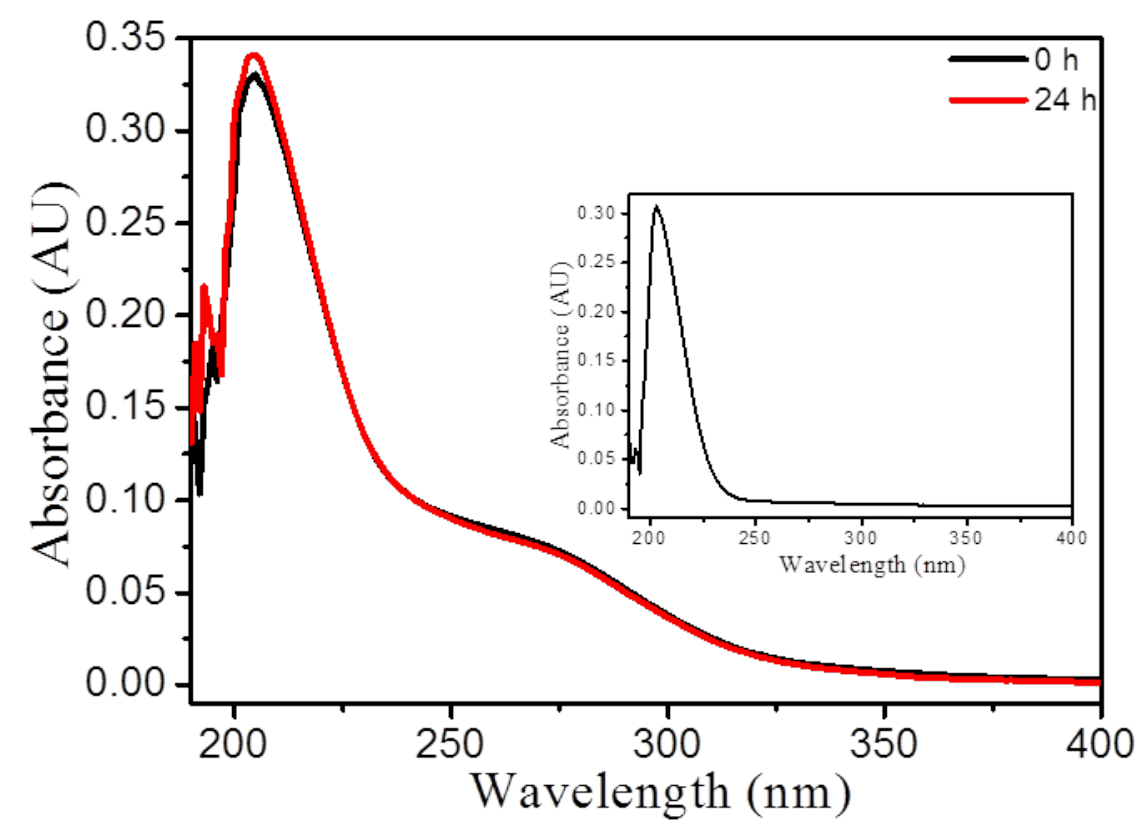

Figure S19. Evolution of the UV spectrum of a solution of POM $4\left(2.5 \times 10^{-6} \mathrm{M}\right)$ with time, recorded in a pH 5 medium (red and black lines). Inset: UV spectrum of a solution of $\mathrm{CoSO}_{4}$ $\left(7.5 \times 10^{-4} \mathrm{M}\right)$ in the same medium.

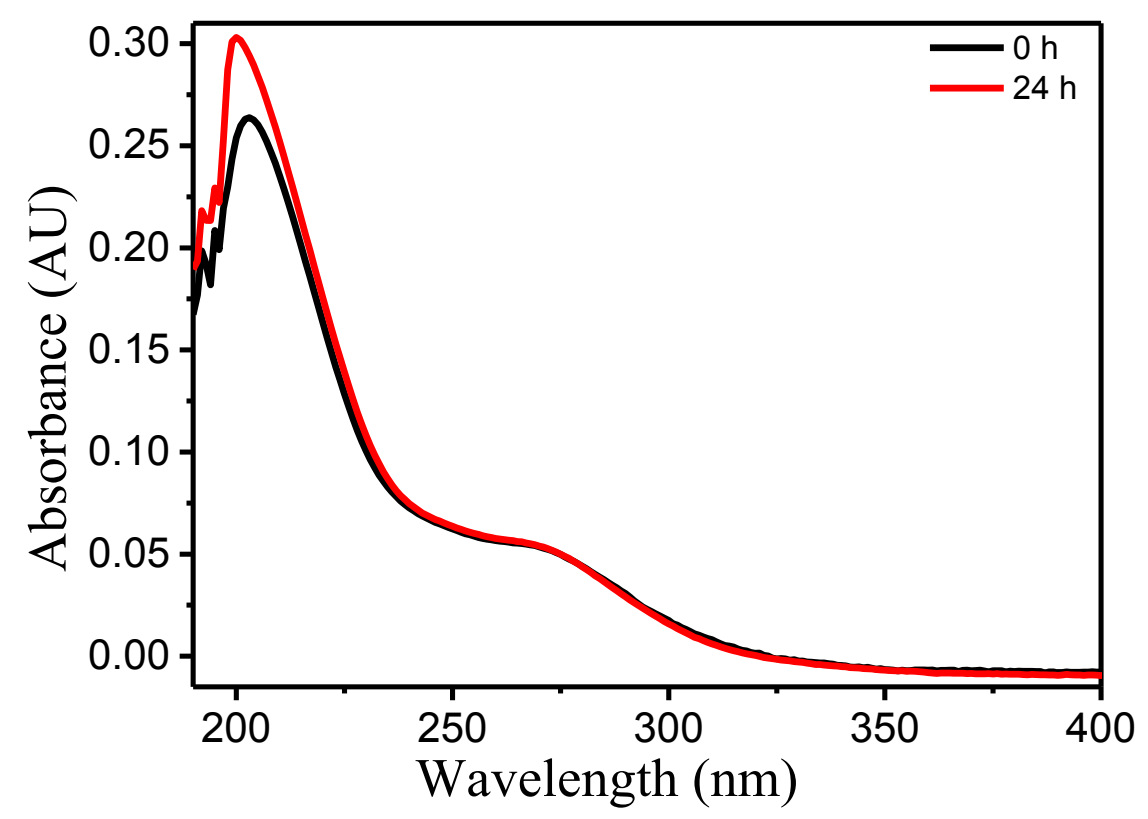

Figure S20. Evolution of the UV spectrum of a solution of POM $4\left(2.5 \times 10^{-6} \mathrm{M}\right)$ with time, recorded in a pH 9 medium (red and black lines). 


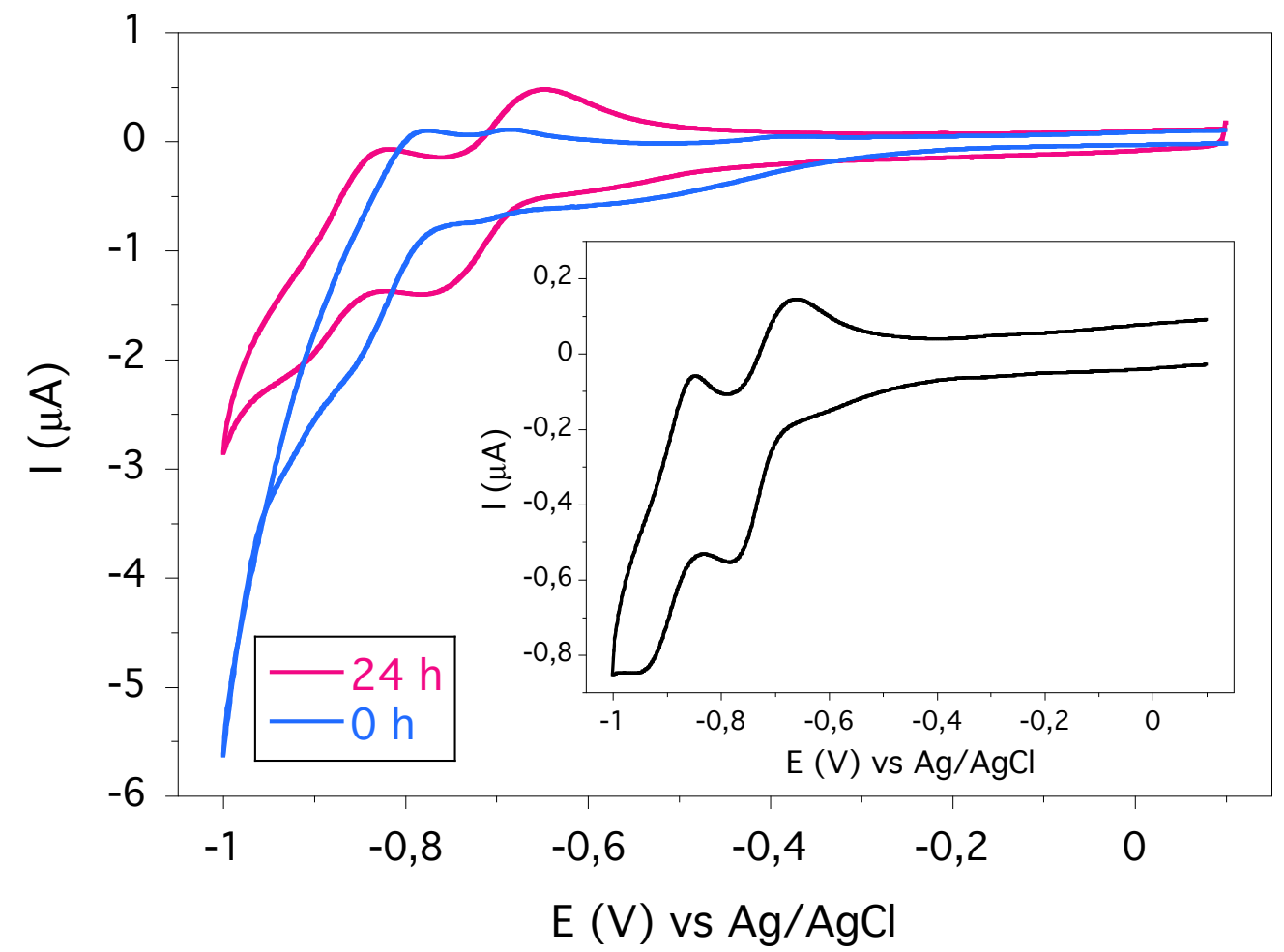

Figure S21. Comparison of an initial cyclic voltammogram of POM $1\left(5 \times 10^{-5} \mathrm{M}\right)$ with the cyclic voltammogram recorded after $24 \mathrm{~h}(\mathrm{pH}=5)$. Inset: cyclic voltammogram of $\left[\mathrm{PCo}\left(\mathrm{H}_{2} \mathrm{O}\right) \mathrm{W}_{11} \mathrm{O}_{39}\right]^{5-}\left(3 \times 10^{-5} \mathrm{M}\right)$ in the same medium.

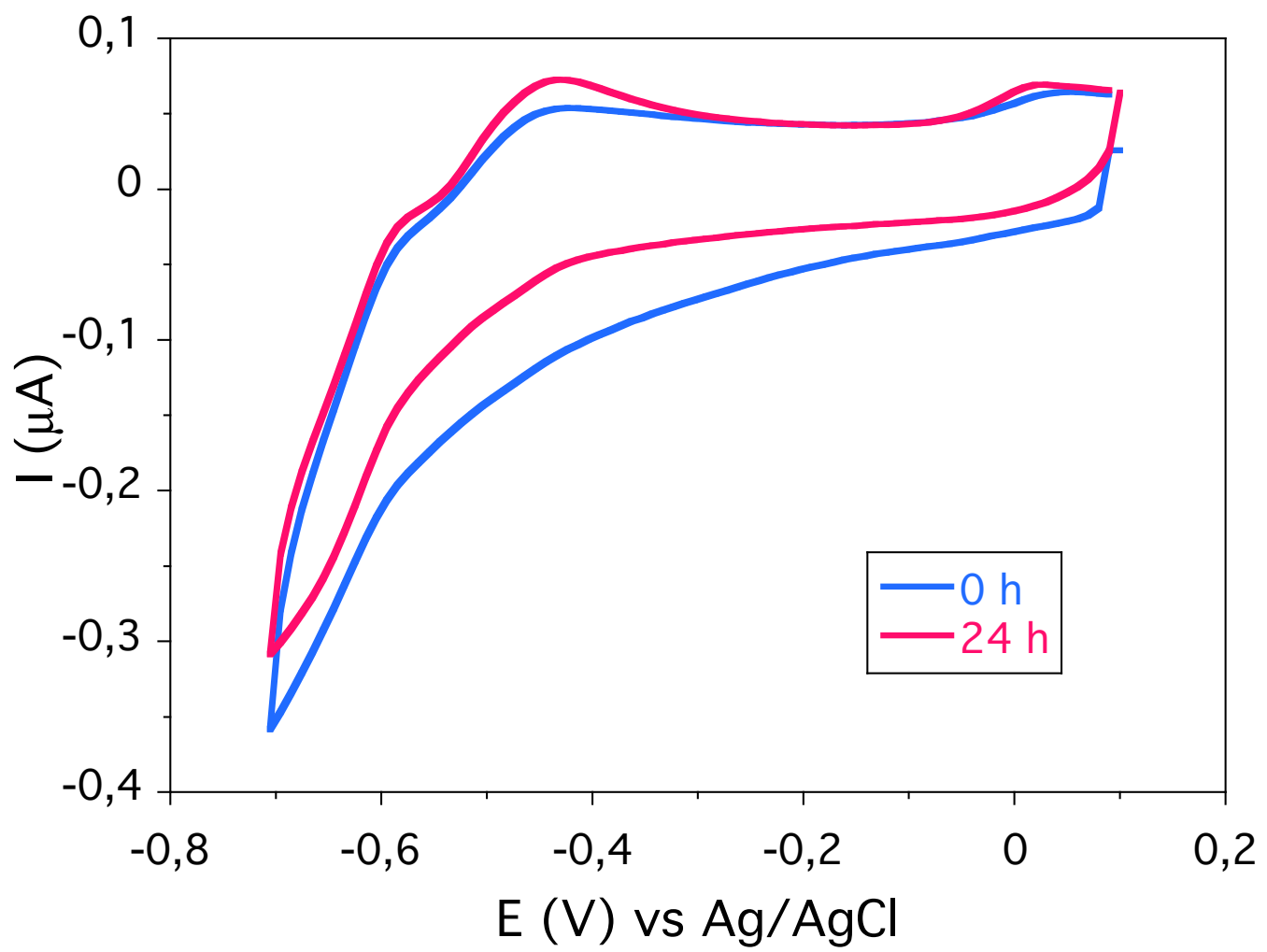

Figure S22. Comparison of an initial cyclic voltammogram of POM $4\left(5 \times 10^{-5} \mathrm{M}\right)$ with the cyclic voltammogram recorded after $24 \mathrm{~h}(\mathrm{pH}=5)$. 


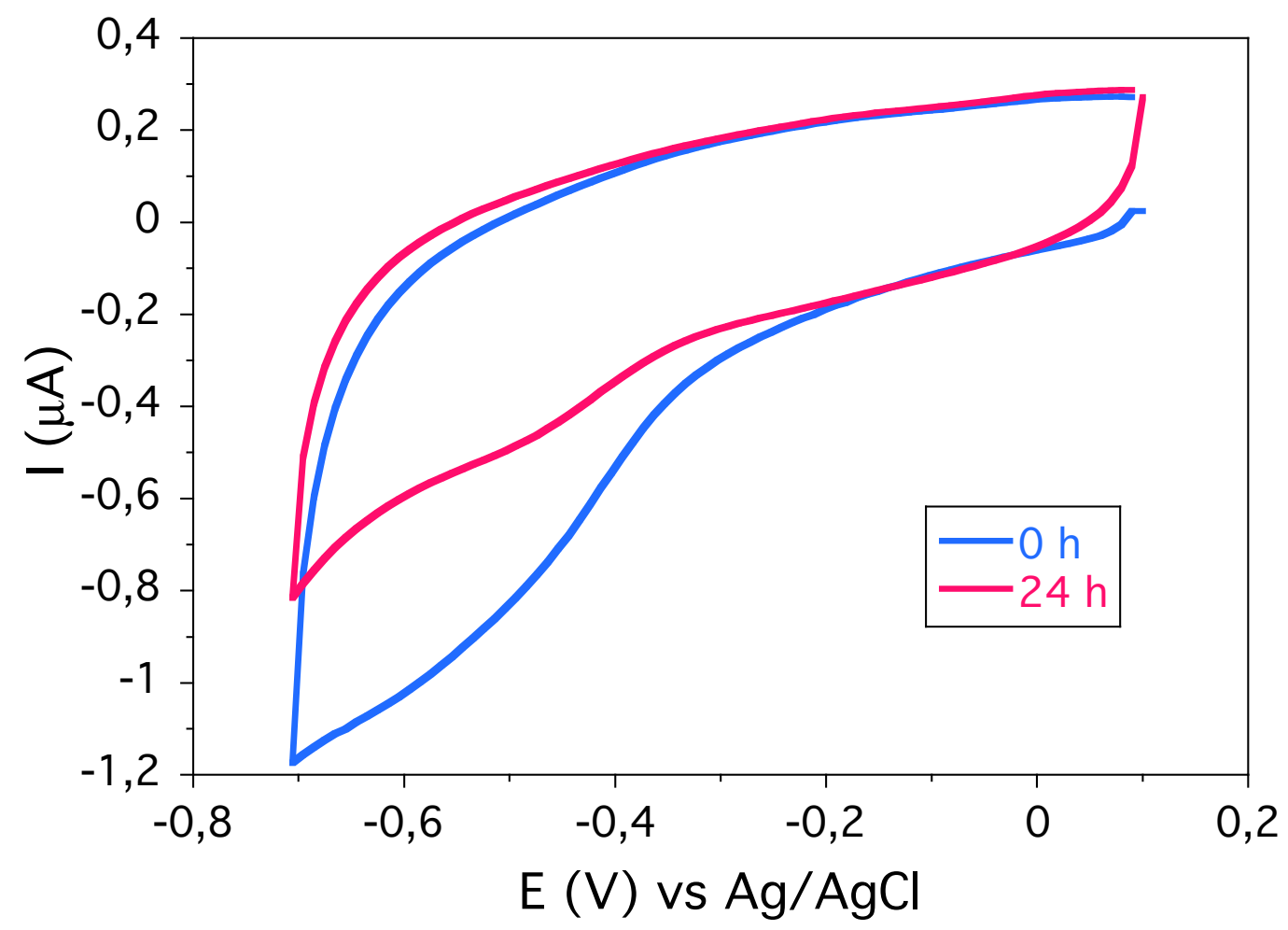

Figure S23. Comparison of an initial cyclic voltammogram of POM $4\left(5 \times 10^{-5} \mathrm{M}\right)$ with the cyclic voltammogram recorded after $24 \mathrm{~h}(\mathrm{pH}=9)$. 